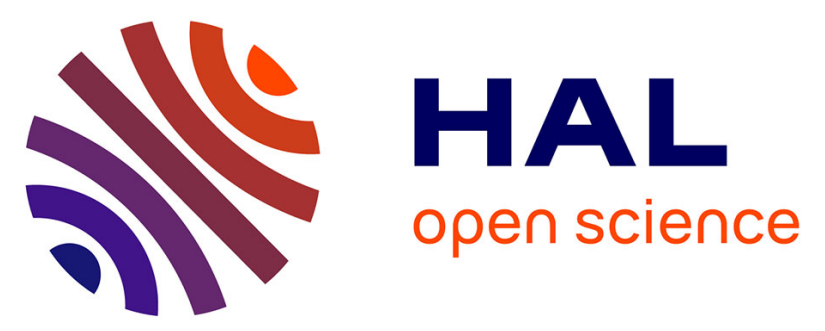

\title{
Natural and anthropogenic particulate-bound aliphatic and polycyclic aromatic hydrocarbons in surface waters of the Gulf of Gabes (Tunisia, southern Mediterranean Sea)
}

Rania Fourati, Marc Tedetti, C. Guigue, M. Goutx, Hatem Zaghden, Sami Sayadi, Boubaker Elleuch

\section{To cite this version:}

Rania Fourati, Marc Tedetti, C. Guigue, M. Goutx, Hatem Zaghden, et al.. Natural and anthropogenic particulate-bound aliphatic and polycyclic aromatic hydrocarbons in surface waters of the Gulf of Gabes (Tunisia, southern Mediterranean Sea). Environmental Science and Pollution Research, 2018, 25 (3), pp.2476-2494. 10.1007/s11356-017-0641-7 . hal-01799490

\section{HAL Id: hal-01799490 \\ https://hal.science/hal-01799490}

Submitted on 27 Feb 2019

HAL is a multi-disciplinary open access archive for the deposit and dissemination of scientific research documents, whether they are published or not. The documents may come from teaching and research institutions in France or abroad, or from public or private research centers.
L'archive ouverte pluridisciplinaire HAL, est destinée au dépôt et à la diffusion de documents scientifiques de niveau recherche, publiés ou non, émanant des établissements d'enseignement et de recherche français ou étrangers, des laboratoires publics ou privés. 




\title{
Natural and anthropogenic particulate-bound aliphatic and polycyclic aromatic hydrocarbons in surface waters of the Gulf of Gabès (Tunisia, southern Mediterranean Sea)
}

\author{
Rania Fourati ${ }^{1}$ - Marc Tedetti ${ }^{2}$ - Catherine Guigue ${ }^{2}$ - Madeleine Goutx ${ }^{2}$. \\ Hatem Zaghden $^{3} \cdot$ Sami Sayadi $^{3}$ - Boubaker Elleuch ${ }^{1}$
}

\begin{abstract}
Particulate-bound aliphatic and polycyclic aromatic hydrocarbons (AHs and PAHs) were investigated in the surface waters of the Gulf of Gabès (Tunisia, southern Mediterranean Sea). Samples were collected off the Sfax and Gabès-Ghannouch coasts. Concentrations in total resolved $n$-alkanes ranged from 0.03 to $3.2 \mu \mathrm{g} \mathrm{L}{ }^{-1}$, and concentrations in total parents + alkylated PAHs ranged from $b d l$ to $108.6 \mathrm{ng} \mathrm{L}^{-1}$. The highest concentrations were recorded in the southern Sfax. AHs were mainly of biogenic origin with odd $n$-alkane predominance, although an anthropogenic contribution was also detected. The PAH molecular patterns revealed a mixed origin with the presence of low molecular weight and alkylated compounds,
\end{abstract}

characteristic of uncombusted oil-derived products, and the presence of high molecular weight compounds, typical of combustion residues. Rainfall events induced an increase in PAH concentrations by a factor 1.5-23.5. The particle-water partition coefficients $\left(\mathrm{K}_{\mathrm{oc}}\right)$ suggest that the partitioning of PAHs between the particulate and dissolved phases is driven by hydrophobicity and organic matter composition.

Keywords Hydrocarbons - Coastal waters · Particles · Organic matter · Partition coefficient · Gulf of Gabès · Mediterranean Sea 


\section{Introduction}

Aliphatic (AHs) and polycyclic aromatic (PAHs) hydrocarbons are among the most investigated hydrocarbon classes in the marine environment. AHs are known to have both biogenic and anthropogenic sources. They can be derived from bacteria, viruses, fungi, zooplankton and phytoplankton, as well as higher plant waxes (Cripps 1989; Rielley et al. 1991; Volkman et al. 1992), but also from unburned petroleum/oil (Bouloubassi and Saliot 1993; Readman et al. 2002; Wang et al. 1997). Although PAHs may have biogenic sources (Chen et al. 1998; Wilcke et al. 2002), they are generally considered to originate exclusively from anthropogenic activities. They are issued from unburned petroleum or oil-derived products (petrogenic PAHs) or from the incomplete combustion of fossil fuels and biomass (pyrogenic PAHs) (Hylland 2006; Neff 2002; Wang et al. 1999).

Petrogenic PAHs are mainly composed of low molecular weight (LMW) two-to-three-ring compounds and their alkylated homologues. They enter marine waters mostly at the dissolved state through different processes, such as airwater gas exchanges, oil spills and sea-based activities (Castro-Jiménez et al. 2012; Tornero and Hanke 2016; Tsapakis et al. 2006). Pyrogenic PAHs include high molecular weight (HMW) four-to-six-ring compounds. They enter marine waters mostly associated with soot and black carbon particles via dry and wet atmospheric depositions, rivers and runoffs (Liu et al. 2012; Shrestha et al. 2010; Tsapakis et al. 2006). Sorption on biogenic (mainly LMW compounds) or atmospheric (mainly HMW compounds) particles and the subsequent downward settling is now recognized as being a major process in the removing of PAH from surface marine waters (Adhikari et al. 2015; Baker et al. 1991; Bouloubassi et al. 2006).

Particulate-bound hydrocarbons may be thus deposited to sediment through vertical sinking (Dachs et al. 2002; Duran and Cravo-Laureau 2016), transferred from sediment to water column via sediment resuspension events (Eggleton and Thomas 2004; Ko et al. 2003), transferred to dissolved water phase from desorption processes (Baker et al. 1991), may undergo photodegradation and biodegradation (Dachs et al. 1999; Shang et al. 2015) or may enter marine food web through ingestion of particles by filter feeders (Akkanen et al. 2012). Overall, the fate and dynamics of hydrocarbons in the water column are largely driven by their partitioning between the particulate and dissolved phases, which is quantified via the particulate-water or particulate organic carbon (POC)-water partition coefficients $\left(\mathrm{K}_{\mathrm{d}}\right.$ and $\mathrm{K}_{\mathrm{oc}}$, respectively) (Herbes 1977).

The Gulf of Gabès, located in the southeast part of Tunisia (southern Mediterranean Sea), is characterized by high marine biodiversity and productivity, which makes it the main fishing area of Tunisia (Bel Hassen et al. 2009; Ben Amor and
Gueddari 2016). However, in recent years, the Gulf of Gabès has been strongly affected by anthropogenic activity with various inputs from coastal urban centres, fishing and commercial harbours, phosphate processing plants and other industries, municipal landfills, wastewater treatment plants (WWTPs) and olive oil waste storage ponds (Alaya-Ltifi and Selmi 2014; Aloulou et al. 2012; Azri et al. 2002; Drira et al. 2016). So far, studies dealing with hydrocarbons in the Gulf of Gabès have mainly focused on surficial sediments (Aloulou et al. 2010; Amorri et al. 2011; Louati et al. 2001; Zaghden et al. 2005, 2007, 2014, 2017).

In this work, we characterized, for the first time, the hydrocarbon (AHs and PAHs) content in the particulate phase of surface waters of the Gulf of Gabès, in the Sfax and GabèsGhannouch coastal areas. In a companion paper, we reported the spatial distribution and sources of dissolved AHs and PAHs (Fourati et al. 2017). As for dissolved hydrocarbons, here we took into consideration several biogeochemical parameters (nutrients, organic matter (OM), chlorophyll) to better understand the dynamics of particulate AHs and PAHs. Our specific objectives are (1) to assess the spatial distribution and identify the potential origins and sources of particulatebound AHs and PAHs, (2) to quantify the partitioning between particulate and dissolved PAHs through $\mathrm{K}_{\mathrm{oc}}$ measurements and attempt to understand factors that control this partitioning and (3) to determine the effect of rainfall events on the distribution of particulate hydrocarbons in this semi-arid, highly anthropogenized environment.

\section{Materials and methods}

\section{Study area}

The study was conducted in coastal areas of the Gulf of Gabès (southeast of Tunisia), i.e. the northern and southern coasts of Sfax city, Mahrès delegation, Kerkennah Islands and GabèsGhannouch area (Fig. 1). Sfax $\left(34^{\circ} 43^{\prime} \mathrm{N}, 10^{\circ} 46^{\prime}\right.$ E) represents the second largest city in Tunisia in terms of population and economic activity. The Sfax northern coast, which extends from the commercial harbour to Ezzit wadi and beyond (Fig. 1), was recently restored thanks to the Taparura project, which aimed to remediate the coast between the commercial harbour and Sidi Mansour affected by past phosphate processing activities. This coast also includes the outlet of the Ezzit wadi, which receives untreated industrial and domestic effluents, the outlet of the rainwater drain channel $\mathrm{PK}_{4}$, which crosses the city from the southwest to the northeast, and the Poudrière industrial zone.

The Sfax southern coast, which extends from the commercial harbour to the southern boundary of the Salina/Thyna agglomeration (Fig. 1), encompasses a phosphate processing plant, the industrial zones of Sidi Salem and Madagascar, the 


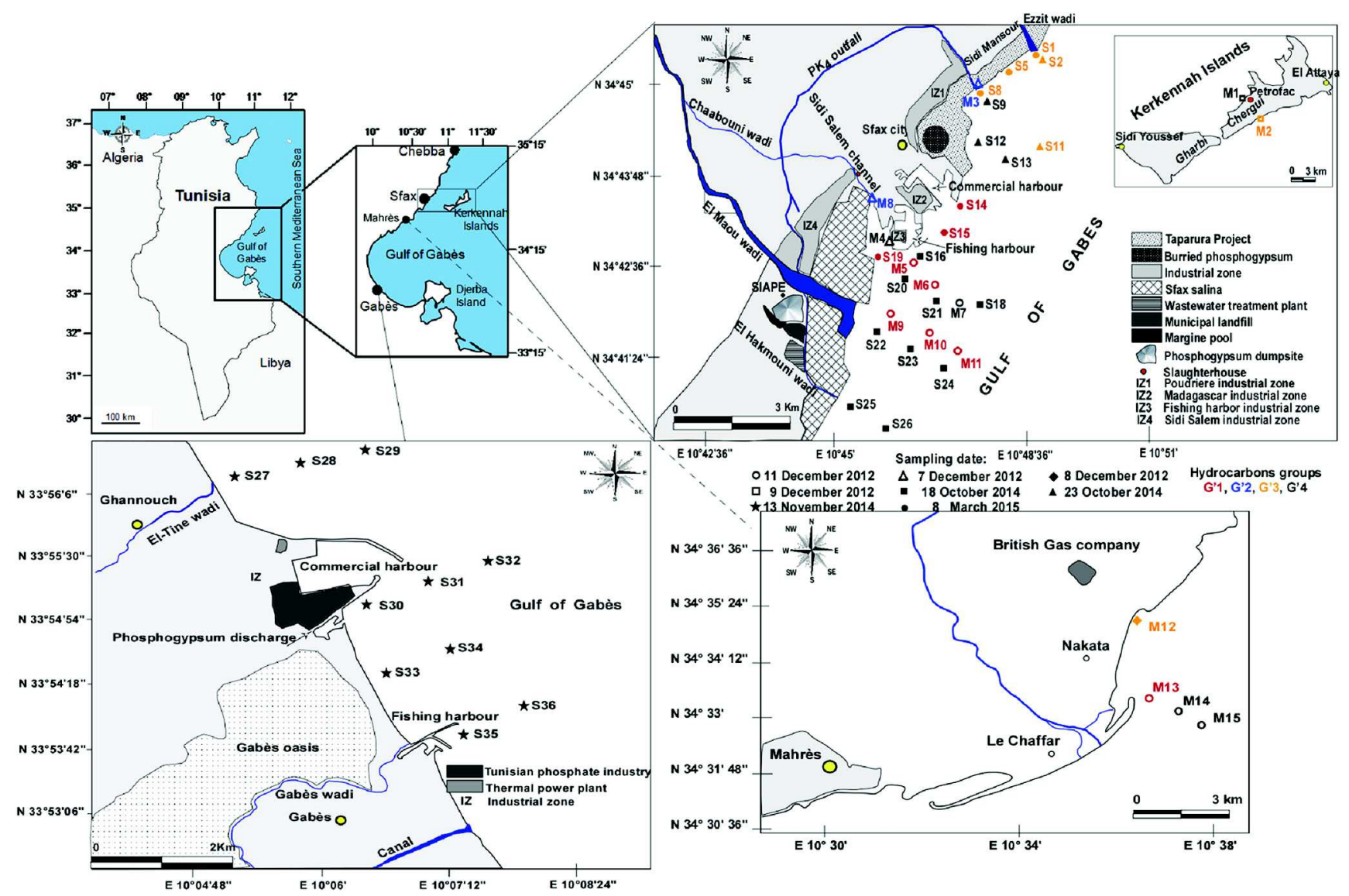

Fig. 1 Location of the study stations in the northern and southern coasts of Sfax city, Mahrès delegation, Kerkennah Islands and GabèsGhannouch area, in the Gulf of Gabès (southeastern Mediterranean Sea, Tunisia). The detailed characteristics of the stations are given in Table S1.

The four groups of stations $\left(\mathrm{G}^{\prime} 1-\mathrm{G}^{\prime} 4\right)$ identified according to the hierarchical ascendant classification applied to the hydrocarbon contents are presented with different colours

fishing and commercial harbours, a wastewater treatment plant (WWTP) and the abandoned Thyna municipal landfill (DGEQV 2008; Fourati et al. 2017). Some industrial effluents, together with the wastewater effluent, are released into the sea through a channel that joins the El Hakmouni wadi eastward of the WWTP. The El Maou wadi also receives diverse wastes from Sidi Salem industries, while the Sidi Salem channel drains into the sea a large part of rainwater from the southern coast and various untreated industrial wastes including those from the Sfax slaughterhouse (DGEQV 2008).

Mahrès delegation lies $30 \mathrm{~km}$ south of Sfax city (Fig. 1) and houses the British Gas plant, which provides more than $60 \%$ of Tunisian domestic gas production. The Kerkennah Islands, which are located $20 \mathrm{~km}$ (offshore) east of the Sfax city at the northern end of the Gulf of Gabès (Fig. 1), are composed of two main islands (Gharbi and Chergui) and marked by a flat relief (<13 m) (Trabelsi et al. 2015, 2016). The islands cover an area of $160 \mathrm{~km}^{2}$ and are characterized by fishing, maritime traffic (transit of ferry and ships) and the PETROFAC gas plant, located in the northwestern part of the Chergui Island ( $<10 \%$ of Tunisia's gas production).

The Gabès-Ghannouch area $\left(33^{\circ} 56^{\prime} \mathrm{N}, 10^{\circ} 03^{\prime} \mathrm{E}\right.$; Fig. 1$)$ is characterized by important harbour and industrial activities. The area houses an important chemical complex with a phosphate processing plant, which directly discharges phosphogypsum into the sea south of the commercial harbour (Alaya-Ltifi and Selmi 2014; Béjaoui et al. 2004). This area also includes a thermal power plant in the northern part of the commercial harbour, a fishing harbour and various industrial activities, such as cement works, petrochemical and offshore petroleum infrastructures, energy production, agribusiness and textile manufactures. Several wadis cover this area, such as the El-Tine and the Gabès wadis, which are used as evacuators of diverse industrial and domestic wastes (Fig. 1).

\section{Sampling}

Forty-nine stations were sampled off the northern and southern coasts of Sfax city, Mahrès delegation, Kerkennah Islands and Gabès-Ghannouch area in December 2012, January 2013, October-November 2014 and March 2015 (details are given in Table S1). Stations M3-AR, M4 $4_{-A R}, M 8_{-A R}$ and M12 $2_{-A R}$ 
were sampled within $24 \mathrm{~h}$ after rainfall events at M3, M4, M8 and M12, respectively. Stations M1-M4, M8, M12, M3 $3_{-\mathrm{AR}}$, M4-AR, M8 ${ }_{-A R}$ and M12-AR were sampled from the coast, while all the other stations were sampled on board the Taparura vessel. This sampling occurred around high tide. Seawater samples were collected at a depth of $\sim 0.1 \mathrm{~m}$ using 4-L Nalgene ${ }^{\circledR}$ polycarbonate bottles, which were opened below the water surface to avoid sampling of the surface microlayer. Before their use, these bottles were washed with $1 \mathrm{M}$ hydrochloric acid $(\mathrm{HCl})$ and ultrapure water (i.e. Milli-Q water from Millipore system, final resistivity $18.2 \mathrm{M} \Omega \mathrm{cm}^{-1}$ ). They were then rinsed with each respective sample before being filled and placed in a cold and dark area after collection, until back to laboratory. On board, in situ measurements of temperature and $\mathrm{pH}$ were carried out using measuring cells with the TetraCon ${ }^{\circledR}$ four-electrode system, while salinity measurements were conducted using a WTW refractometer (Weilheim, Germany).

\section{Filtration, handling and storage of samples}

In the laboratory, samples were immediately filtered under low vacuum conditions $(<50 \mathrm{mmHg})$ through precombusted $\left(500{ }^{\circ} \mathrm{C}, 4 \mathrm{~h}\right) \mathrm{GF} / \mathrm{F}(\sim 0.7-\mu \mathrm{m})$ glass fibre filters ( 25 or $47 \mathrm{~mm}$ diameter, Whatman) using glassware filtration systems. Particulate hydrocarbons (AHs and PAHs) were recovered onto 47-mm-diameter GF/F filters. Filtered volume comprised between 0.5 and $2 \mathrm{~L}$ depending on stations. Filters were then stored in Nunc ${ }^{\circledR}$ cryogenic vials at $-20{ }^{\circ} \mathrm{C}$ until analysis (see "Hydrocarbons extraction, purification and instrumental analysis" section). It should be noted that filtered seawater was also used for dissolved $\mathrm{AH}$ and $\mathrm{PAH}$ analyses (results presented in Fourati et al. 2017). In addition, from filtered seawater and filters, several biogeochemical parameters were analysed for stations S2, S9, S11-S13, S16, S18 and S20-S36, i.e. total chlorophyll $a$ (TChl $a)$, suspended particulate matter (SPM), particulate organic carbon (POC) and nitrogen (PON), dissolved organic carbon (DOC) and nutrients: nitrates $\left(\mathrm{NO}_{3}{ }^{-}\right)$, nitrites $\left(\mathrm{NO}_{2}{ }^{-}\right)$, phosphates $\left(\mathrm{PO}_{4}{ }^{3-}\right)$ and silicates $\left[\mathrm{Si}\left(\mathrm{OH}_{4}\right)\right]$. Detail of these biogeochemical analyses is provided in Fourati et al. (2017).

\section{Hydrocarbons extraction, purification and instrumental analysis}

Particulate hydrocarbons on the filters (fraction $\geq 0.7 \mu \mathrm{m}$ ) were extracted using Bligh and Dyer's (1959) method for stations M12-M15, M3 ${ }_{-A R}, M 4_{-A R}, M 8_{-A R}$ and M12 ${ }_{-A R}$, and using Accelerated Solvent Extraction (Dionex ASE 350) technique for stations M1-M11 and S1-S36. The extraction using Bligh and Dyer's method consists in adding a monophasic solvent mixture [dichloromethane $\left(\mathrm{CH}_{2} \mathrm{Cl}_{2}\right) /$ methanol $\left.\left(\mathrm{CH}_{3} \mathrm{OH}\right) / \mathrm{H}_{2} \mathrm{O} ; 1 / 2 / 0.8 ; v / v / v\right]$ to filters which were cut into small pieces. This solvent mixture was sonicated $(2 \times 8 \mathrm{~min})$ then filtered, and a biphasic mixture $\left.\left[\mathrm{CH}_{2} \mathrm{Cl}_{2} / \mathrm{H}_{2} \mathrm{O} ; 1 / 1 ; v / v\right)\right]$ was added to recover the hydrophobic compounds in the organic phase. For the ASE extraction technique, filters were extracted in a 5-mL stainless-steel cell using $\mathrm{CH}_{2} \mathrm{Cl}_{2}$ as solvent. The following ASE parameters were used: system pressure at $10 \mathrm{MPa}(1500 \mathrm{psi})$, oven temperature of $150{ }^{\circ} \mathrm{C}$ with heat up time of $7 \mathrm{~min}$, static time of $10 \mathrm{~min}, 3$ static cycles, rinsing volume: $100 \%$ of extraction volume and nitrogen purge of $60 \mathrm{~s}$. The extracted analytes were then collected in vial inserts. Next, the analytic steps were similar for both extraction methods (Bligh and Dyer and ASE). Extracts were concentrated by using a rotary evaporator, and solvent was changed to $n$-hexane. Hexane extracts were then purified to separate the hydrocarbon fractions from more polar compounds by fractioning them within a 500-mg silica column (Silica gel extra pure, Merck), which had previously been activated at $500{ }^{\circ} \mathrm{C}$ for $4 \mathrm{~h}$ and then partially deactivated with ultrapure water $(4 \% w / w)$. AHs and PAHs were eluted successively using $2 \mathrm{~mL}$ of $n$-hexane and a mixture of $3 \mathrm{~mL}$ of $n$ hexane $/ \mathrm{CH}_{2} \mathrm{Cl}_{2}(3: 1 ; v / v)$, respectively (Guigue et al. 2015). All solvents were of organic trace analysis quality (Rathburn Chemicals Ltd).

Purified hydrocarbon fractions (AHs and PAHs) were analysed by a gas chromatograph-mass spectrometer (GCMS) (TraceISQ, ThermoElectron, GC ultra and 5977A Series GC/MSD System, Agilent Technologies) running at an ionisation energy of $70 \mathrm{eV}$ for a $\mathrm{m} / \mathrm{z}$, range of 50-400, and using simultaneously the full scan and the selected ion monitoring (SIM) modes for TraceISQ GC and only the SIM mode for Agilent GC. The GC-MS used helium at a flow rate of $1.2 \mathrm{~mL} \mathrm{~min}^{-1}$ as a carrier gas and an HP-5 MS column $(30 \mathrm{~m} \times 0.25 \mathrm{~mm} \times 0.25 \mu \mathrm{m}, \mathrm{J} \& \mathrm{~W}$ Scientific, Agilent Technologies). The injector (used in splitless mode) and detector temperatures were set at 270 and $320^{\circ} \mathrm{C}$, respectively. The non-linear temperature program used for the column was as follows: $70^{\circ} \mathrm{C}$ for $3 \mathrm{~min}, 15^{\circ} \mathrm{C} \mathrm{min}^{-1}$ (ramp 1) to $150^{\circ} \mathrm{C}$ and then $7^{\circ} \mathrm{C} \mathrm{min}^{-1}$ (ramp 2) to a final temperature of $320^{\circ} \mathrm{C}$, held for $10 \mathrm{~min}$. The identification and quantification of hydrocarbons in samples and calibrations were performed in SIM mode (Guigue et al. 2015).

\section{Quality assurance/quality control}

$\mathrm{AH}$ and PAH external calibration standards (16 parent PAHs and 35 AHs, 04071, Fluka and 47543-U, Supelco, purchased from Sigma-Aldrich) were run for the peak identification and the evaluation of response factors between compounds. Deuterated standard mixtures were introduced in samples prior to extraction ( $n-\mathrm{C}_{16}-d_{34}, n-\mathrm{C}_{24}-d_{50}, n-\mathrm{C}_{36}-d_{74}$ for AHs, and Naph- $d_{8}$, Phe- $d_{10}$, Per- $d_{12}$ for PAHs) and prior to injection (Ace- $d_{10}$, Flu- $d_{10}$ and Chrys- $d_{12}$ ) in order to determine procedural recoveries and quantify hydrocarbons in samples. 
Average recoveries of $n-\mathrm{C}_{16}-d_{34}, n-\mathrm{C}_{24}-d_{50}$ and $n-\mathrm{C}_{36}-d_{74}$ were 74,82 and $79 \%$, respectively, and those of Naph- $d_{8}$, Phe- $d_{10}$, Per- $d_{12}$ were 50, 68 and $78 \%$, respectively. Blanks were run for the entire procedure including extraction, solvent concentration and purification. All concentration values were blank- and recovery-corrected. Detection limits of the method used for individual compounds varied from 1 to $30 \mathrm{pg} \mathrm{L}^{-1}$.

\section{Determination of hydrocarbon indices and ratios}

For AHs, we determined the concentration of resolved compounds $(R)$, i.e. $n$-alkanes from $n-\mathrm{C}_{15}$ to $n$ - $\mathrm{C}_{40}$, including pristane (Pr) and phytane (Phy), and the concentration of the unresolved complex mixture (UCM). AH molecular pattern distributions were examined in conjunction with several ratios and indices to distinguish biogenic from anthropogenic AHs. First, we used the carbon preference index $\left(\mathrm{CPI}_{15-40}\right)$, computed as the odd/even ratio in the $n-\mathrm{C}_{15}-n-\mathrm{C}_{40}$ range, as an indicator of biogenic AHs $\left(\mathrm{CPI}_{15-40} \geq 2\right.$ or $\left.<1\right)$ or AHs issued from petroleum contaminations $\left(\mathrm{CPI}_{15-40} \sim 1\right)$ (Commendatore et al. 2012). Second, we used the Pr/Phy ratio as an indicator of biogenic AHs (when > 1) (Cincinelli et al. 2008; Commendatore and Esteves 2004; Cripps 1989). Due to the controversial origin of these isoprenoids, we did not use this ratio to highlight the possible petrogenic origin of $\mathrm{AHs}$ (when it is < 1). Finally, we modified the terrigenous/aquatic ratio (Ter/aq), used by Bourbonniere and Meyers (1996) and Mille et al. (2007), to give a better fit to our molecular profile distributions. This ratio was calculated as the concentrations of long-chain $n$-alkanes $\left(n-\mathrm{C}_{29}+n-\mathrm{C}_{31}+n-\mathrm{C}_{33}\right)$ to short-chain $n$-alkanes $\left(n-\mathrm{C}_{15}+n-\mathrm{C}_{17}+n-\mathrm{C}_{19}\right)$, as an index of the relative importance of terrestrial (higher plants; Ter/aq $>1$ ) and aquatic (algae, phytoplankton and zooplankton; Ter/aq < 1) materials.

We quantified $29 \mathrm{PAH}$ compounds $\left(\sum_{29} \mathrm{PAHs}_{-\mathrm{P}+\mathrm{A}}\right)$ in these samples: 19 parent PAHs (PAHs_P) and 10 alkylated homologues (PAHs-A). PAHs $-\mathrm{P}$ include naphthalene (Naph), acenaphthylene (Acy), acenaphthene (Ace), fluorene (Flu), dibenzothiophene (DBT), phenanthrene (Phe), anthracene (Ant), fluoranthene (Flt), pyrene (Pyr), benz[a]anthracene [BaA], chrysene ( $\mathrm{Chr}$, benzo[b]fluoranthene [BbF], benzo[k]fluoranthene [BkF], benzo[a]pyrene [BaP], benzo[e]pyrene [BeP], perylene (Per), dibenzo[a,h]anthracene [DA], benzo[g,h,i]perylene [BP] and indeno[1,2,3-cd]pyrene [IP]. PAHs $-\mathrm{A}$ comprise methyl $\left(\mathrm{C}_{1}\right)$, dimethyl $\left(\mathrm{C}_{2}\right)$ and trimethyl $\left(\mathrm{C}_{3}\right)$ of Naph and Phe and $\mathrm{C}_{1}$ and $\mathrm{C}_{2}$ of Flu and Pyr. Several ratios were used to discriminate petrogenic and pyrogenic PAHs (Budzinski et al. 1997; Lipiatou and Saliot 1991; Mille et al. 2007). First, we used the ratio between alkylated homologues and parent compounds (Alk/Par), and the ratio between LMW and HMW compounds (LMW/ HMW $_{-\mathrm{P}+\mathrm{A}}$ ). Petrogenic PAHs are rather characterized by the dominance of alkylated homologues over their parent compounds and the dominance of LMW (two to three rings) over
HMW (four to six rings) compounds (Alk/Par > 1; LMW/ $\mathrm{HMW}_{-\mathrm{P}+\mathrm{A}}>1$ ), whereas the inverse pattern is observed for pyrogenic PAHs (Alk/Par < 1; LMW/HMW -P+A $<1$ ) (Youngblood and Blumer 1975). Second, we used the Flt/ (Flt + Pyr) ratio, as an indicator of pyrogenic PAHs (grass, coal and wood combustion) ( $>0.5)$, petroleum combustion (0.4-0.5) or petrogenic PAHs $(<0.4)$ (Asia et al. 2009; Li et al. 2015; Yunker et al. 2002). It should be mentioned that all these $\mathrm{AH}$ and $\mathrm{PAH}$ ratios and indices have to be used with precaution given the multiplicity of hydrocarbon sources and transformation processes they have undergone (Katsoyiannis et al. 2007; Katsoyiannis and Breivik 2014; Tobiszewski and Namieśnik 2012).

\section{Statistical treatment}

Hierarchical ascendant classification (HAC) based on Ward's method (squared Euclidean distance) was applied on selected hydrocarbon parameters $\left(R, \mathrm{CPI}_{15-40}\right.$ and Ter/aq for AHs, $\sum_{29}$ $\mathrm{PAHs}_{-\mathrm{P}+\mathrm{A}}$, Alk/Par and LMW/HMW ${ }_{-\mathrm{P}+\mathrm{A}}$ for PAHs). Clusters issued from this HAC were represented by box-and-whisker plots. Principal component analysis (PCA) based on Spearman's rank-order correlation matrix was also applied to these hydrocarbon variables. All tests and analyses were performed with XLSTAT 2013.5.01.

\section{Results}

\section{Concentration levels and spatial distribution of particulate hydrocarbons}

Particulate total $\mathrm{AH}$ and $\mathrm{PAH}$ concentrations and ratios are reported in Table 1. HAC analysis reveals the presence of four groups of stations ( $\mathrm{G}^{\prime} 1, \mathrm{G}^{\prime} 2, \mathrm{G}^{\prime} 3$ and $\left.\mathrm{G}^{\prime} 4\right)$ according to their concentrations and ratios. These groups are represented by PCA (Fig. 2), in the form of box-and-whisker plots (Fig. 3) and colour maps (Figs. S1-S3). AH and PAH molecular profiles are shown in Figs. 4, 5, 6, and 7. Below, we describe the hydrocarbon content of each of these four HAC/PCA groups of stations. It is worth noting that UCM was not detected for these different stations.

\section{Group $G^{\prime} 1$}

G'1 includes stations S14, S15, M5, M6, S19 and M9-M11 from the southern coast of Sfax and M13 from the Mahrès delegation (Fig. 2). This group of stations is characterized by relatively high $R$ concentrations, including Pr and Phy, compared to the rest of the stations, with concentrations varying from 0.31 (S14) to $3.2 \mu \mathrm{g} \mathrm{L}^{-1}$ (M13) (mean $1.1 \pm 1 \mu \mathrm{g} \mathrm{L}^{-1}$ ) (Table 1; Fig. 3a, Fig. S1a). These stations are marked by the dominance of LMW compounds in the $n-\mathrm{C}_{15}-n-\mathrm{C}_{24}$ range and 
Table 1 Particulate aliphatic (AHs) and polycyclic aromatic (PAHs) hydrocarbons: concentrations and ratios

\begin{tabular}{|c|c|c|c|c|c|c|c|c|c|c|c|c|c|}
\hline Stations & $\begin{array}{l}R \\
\left(\mu \mathrm{g} \mathrm{L}^{-1}\right)\end{array}$ & $\mathrm{CPI}_{15-40}$ & $\begin{array}{l}\mathrm{Pr} / \\
\mathrm{Phy}\end{array}$ & $\begin{array}{l}\text { Ter/ } \\
\text { aq }\end{array}$ & $\mathrm{MH}$ & $\begin{array}{l}\sum_{16} \\
\text { PAHs } \\
\left(\mathrm{ng} \mathrm{L}^{-1}\right)\end{array}$ & $\begin{array}{l}\sum_{19} \\
\text { PAHs }_{-\mathrm{P}} \\
\left(\mathrm{ng} \mathrm{L}^{-1}\right)\end{array}$ & $\begin{array}{l}\sum_{29} \mathrm{PAHs}_{-} \\
\mathrm{P}+\mathrm{A} \\
\left(\mathrm{ng} \mathrm{L}^{-1}\right)\end{array}$ & $\begin{array}{l}\text { LMW/ } \\
\text { HMW }_{\mathrm{P}+\mathrm{A}}\end{array}$ & $\begin{array}{l}\text { Alk/ } \\
\text { Par }\end{array}$ & $\begin{array}{l}\text { Flt/ } \\
(\mathrm{Pyr}+\text { Flt })\end{array}$ & $\begin{array}{l}\mathrm{LMW}_{\mathrm{P}+\mathrm{A}} \\
(\%)\end{array}$ & $\begin{array}{l}\mathrm{HMW}_{\mathrm{P}+\mathrm{A}} \\
(\%)\end{array}$ \\
\hline M1 & 0.41 & 11.0 & 0.32 & 0.16 & $n-\mathrm{C}_{17}$ & 0.62 & 0.80 & 3.70 & 14.3 & 4.86 & 0.93 & 93.45 & 6.55 \\
\hline M2 & 0.03 & 3.31 & 1.70 & 0.71 & $n-\mathrm{C}_{15} / n-\mathrm{C}_{31}$ & 0.43 & 0.52 & 1.94 & 21 & 3.42 & 1.00 & 95.41 & 4.59 \\
\hline S1 & 0.13 & 1.50 & 0.46 & 0.72 & $n-\mathrm{C}_{20} / n-\mathrm{C}_{31}$ & 20.0 & 26.0 & 34.5 & 0.50 & 0.89 & 0.48 & 33.27 & 66.73 \\
\hline $\mathrm{S} 2$ & 0.29 & 4.72 & 3.70 & 1.12 & $n-\mathrm{C}_{15} / n-\mathrm{C}_{31}$ & 4.98 & 6.29 & 11.6 & 5 & 1.03 & 0.46 & 83.03 & 16.97 \\
\hline S5 & 0.06 & 5.34 & $n d$ & 0.72 & $n-\mathrm{C}_{15} / n-\mathrm{C}_{31}$ & 0.75 & 0.97 & 2.34 & 3.18 & 2.23 & 0.55 & 76.07 & 23.93 \\
\hline M3 & 0.10 & 2.04 & 0.95 & 0.40 & $n-\mathrm{C}_{15}$ & 0.64 & 0.83 & 2.30 & 9.32 & 2.14 & 0.63 & 90.31 & 9.69 \\
\hline M3 ${ }_{-A R}$ & 2.36 & 1.44 & 0.61 & 0.86 & $n-\mathrm{C}_{17} / n-\mathrm{C}_{31}$ & 5.85 & 9.60 & 51.21 & 3.83 & 8.6 & 0.44 & 79.28 & 20.72 \\
\hline S8 & 0.10 & 3.45 & 0.31 & 0.40 & $n-\mathrm{C}_{15} / n-\mathrm{C}_{31}$ & 2.87 & 4.00 & 6.63 & 0.94 & 2.01 & 0.48 & 48.42 & 52 \\
\hline S9 & 0.27 & 6.15 & 6.00 & 0.42 & $n-\mathrm{C}_{15}$ & 1.07 & 1.11 & 2.07 & 25.7 & 0.94 & 0.61 & 96.25 & 3.75 \\
\hline S11 & 0.22 & 7.93 & 22.5 & 1.80 & $n-\mathrm{C}_{31} / n-\mathrm{C}_{15}$ & 0.53 & 0.62 & 1.04 & 2 & 1.11 & 0.48 & 64.55 & 35.45 \\
\hline S12 & 0.22 & 21.0 & 29.5 & 0.08 & $n-\mathrm{C}_{15}$ & 0.70 & 0.95 & 1.75 & 43.4 & 0.87 & 0.40 & 97.75 & 2.25 \\
\hline S13 & 0.11 & 11.6 & 2935 & 0.05 & $n-\mathrm{C}_{15}$ & 0.03 & 0.04 & 0.27 & 9.13 & 12.92 & nd & 90.13 & 9.87 \\
\hline S14 & 0.31 & 1.79 & 0.89 & 0.05 & $n-\mathrm{C}_{15}$ & 1.47 & 1.70 & 3.67 & 13.9 & 1.26 & 0.49 & 93.29 & 6.71 \\
\hline S15 & 0.47 & 1.19 & 0.72 & 0.04 & $n-\mathrm{C}_{15} / \mathrm{Phy}$ & 2.07 & 2.37 & 8.19 & 11.8 & 2.65 & 0.21 & 92.17 & 7.83 \\
\hline S16 & 0.18 & 8.32 & 11.6 & 0.31 & $n-\mathrm{C}_{15} / n-\mathrm{C}_{31}$ & 0.75 & 0.87 & 2.08 & 13.1 & 1.91 & nd & 92.92 & 7.08 \\
\hline S18 & 0.22 & 9.46 & 1.70 & 0.01 & $n-C_{15}$ & 1.19 & 1.52 & 3.20 & 4.45 & 2.02 & 0.46 & 81.65 & 18.35 \\
\hline M4 & 0.07 & 4.92 & 6.61 & 0.11 & $n-\mathrm{C}_{15}$ & 1.19 & 1.79 & 2.75 & 6.65 & 0.67 & 0.76 & 86.93 & 13.07 \\
\hline M4 $4_{-A R}$ & 1.31 & 1.59 & 0.82 & 0.16 & $n-\mathrm{C}_{17}$ & 3.83 & 4.69 & 20.8 & 2.59 & 6.78 & 0.46 & 72.15 & 27.85 \\
\hline M5 & 0.74 & 1.40 & 1.27 & 0.01 & $n-\mathrm{C}_{15} / n-\mathrm{C}_{17}$ & 0.95 & 1.53 & 6.23 & 6.07 & 4.17 & 0.41 & 85.85 & 14.15 \\
\hline M6 & 0.85 & 1.57 & 1.19 & 0.03 & $n-\mathrm{C}_{15}$ & 1.11 & 2.04 & 6.49 & 5.51 & 3.46 & 0.50 & 84.65 & 15.35 \\
\hline M7 & 0.10 & 5.50 & 2.81 & 0.003 & $n-\mathrm{C}_{15}$ & 0.63 & 0.70 & 1.79 & 10.6 & 2.08 & nd & 91.34 & 8.66 \\
\hline M8 & 1.62 & 1.97 & 0.68 & 0.27 & $n-\mathrm{C}_{17}$ & 9.33 & 13.7 & 70.6 & 7.15 & 6.08 & 0.41 & 87.73 & 12.27 \\
\hline$M 8_{-A R}$ & 3.24 & 2.25 & 1.14 & 0.37 & $n-\mathrm{C}_{17} / n-\mathrm{C}_{29}$ & 8.29 & 13.1 & 108.6 & 9.05 & 9.19 & 0.36 & 90.05 & 9.95 \\
\hline S19 & 0.42 & 1.50 & 0.74 & 0.07 & $n-\mathrm{C}_{15} / n-\mathrm{C}_{17}$ & 2.00 & 2.51 & 9.17 & 9.12 & 2.99 & 0.46 & 90.12 & 9.88 \\
\hline S20 & 0.16 & 6.86 & 10.2 & 0.22 & $n-\mathrm{C}_{15} / n-\mathrm{C}_{31}$ & 2.48 & 3.73 & 5.19 & 1.96 & 0.70 & 0.41 & 66.21 & 33.79 \\
\hline S21 & 0.27 & 36.0 & 39.6 & 0.01 & $n-\mathrm{C}_{15}$ & 1.16 & 2.15 & 2.94 & 4.13 & 0.48 & 0.39 & 80.49 & 19.51 \\
\hline M9 & 2.50 & 1.20 & 1.17 & 0.01 & $n-\mathrm{C}_{17}$ & 1.12 & 2.00 & 12.5 & 7.87 & 7.04 & 0.37 & 88.72 & 11.28 \\
\hline M10 & 0.65 & 1.33 & 1.25 & 0.002 & $n-\mathrm{C}_{15}$ & 1.25 & 1.30 & 5.41 & 11.4 & 3.78 & 0.23 & 91.94 & 8.06 \\
\hline M11 & 0.64 & 1.52 & 1.22 & 0.05 & $n-\mathrm{C}_{15}$ & 1.68 & 2.14 & 6.52 & 18.5 & 2.18 & 0.38 & 94.86 & 5.14 \\
\hline S22 & 0.19 & 5.46 & 2.53 & 0.10 & $n-\mathrm{C}_{15}$ & 1.45 & 2.18 & 4.66 & 2.28 & 3.90 & 0.36 & 69.52 & 30.48 \\
\hline S23 & 0.36 & 25.0 & 5.61 & $n d$ & $n-\mathrm{C}_{15}$ & 2.21 & 2.73 & 4.66 & 1.50 & 2.09 & 0.34 & 59.96 & 40.04 \\
\hline S24 & 0.13 & 135 & $n d$ & 0.01 & $n-\mathrm{C}_{15}$ & 0.41 & 0.47 & 0.67 & 0.91 & 2.09 & nd & 47.61 & 52.39 \\
\hline S25 & 0.22 & 19.6 & nd & 0.08 & $n-\mathrm{C}_{15}$ & 0.24 & 0.41 & 0.97 & 2.43 & 4.78 & 0.36 & 70.87 & 29.13 \\
\hline S26 & 0.25 & 30.8 & $n d$ & 0.02 & $n-\mathrm{C}_{15}$ & 0.27 & 0.38 & 4.26 & 22.9 & 20.25 & nd & 95.81 & 4.19 \\
\hline M12 & 0.08 & 3.62 & 0.34 & 0.88 & $n-\mathrm{C}_{15} / n-\mathrm{C}_{31}$ & 0.29 & 0.40 & 0.7 & 1 & 1.47 & 0.57 & 50.94 & 49.06 \\
\hline M12 & 0.29 & 4.65 & 0.18 & 1.11 & $n-\mathrm{C}_{15} / n-\mathrm{C}_{31}$ & 1.36 & 1.47 & 2.10 & 1 & 0.97 & 0.45 & 49.35 & 50.65 \\
\hline M13 & 3.20 & 1.15 & 1.24 & 0.001 & $n-\mathrm{C}_{17}$ & 0.90 & 1.27 & 11.9 & 7.92 & 12.6 & 0.38 & 88.79 & 11.21 \\
\hline M14 & 0.14 & 36.0 & $n d$ & 0.04 & $n-\mathrm{C}_{15}$ & 0.23 & 0.23 & 0.32 & 1.34 & 0.63 & 0.54 & 57.20 & 42.80 \\
\hline M15 & 0.21 & 5.75 & 2.37 & 0.02 & $n-\mathrm{C}_{15}$ & 0.23 & 0.27 & 0.43 & 0.92 & 1.21 & 0.56 & 48.01 & 51.99 \\
\hline S27 & 0.15 & 6.73 & 7.84 & 0.07 & $n-\mathrm{C}_{15}$ & 0.52 & 0.52 & 0.97 & 4.77 & 0.91 & nd & 82.67 & 17.33 \\
\hline S28 & 0.18 & 4.28 & 31.8 & 0.14 & $n-\mathrm{C}_{15}$ & 0.06 & 0.06 & 0.17 & 0.71 & 1.83 & nd & 41.42 & 58.58 \\
\hline S29 & 0.15 & 5.55 & 6.00 & 0.11 & $n-\mathrm{C}_{15}$ & $b d l$ & $b d l$ & $b d l$ & $n d$ & nd & nd & nd & $n d$ \\
\hline S30 & 0.17 & 4.47 & 6.50 & 0.10 & $n-\mathrm{C}_{15}$ & 0.92 & 1.00 & 2.25 & 1.70 & 1.35 & 0.12 & 63.00 & 37.00 \\
\hline S31 & 0.15 & 5.22 & 12.0 & 0.10 & $n-\mathrm{C}_{15}$ & 0.21 & 0.21 & 0.45 & 0.52 & 1.18 & 0.08 & 34.30 & 65.70 \\
\hline S32 & 0.23 & 3.14 & 2.25 & 0.09 & $n-\mathrm{C}_{15}$ & 0.43 & 0.46 & 2.16 & 1.94 & 4.38 & 0.08 & 65.95 & 34.05 \\
\hline S33 & 0.19 & 4.48 & 6.30 & 0.08 & $n-\mathrm{C}_{15}$ & 0.62 & 0.76 & 2.70 & 0.52 & 2.99 & 0.06 & 34.32 & 65.68 \\
\hline S34 & 0.23 & 3.07 & 3.64 & 0.11 & $n-\mathrm{C}_{15}$ & 1.34 & 1.70 & 6.50 & 1.20 & 3.85 & 0.04 & 54.45 & 45.55 \\
\hline
\end{tabular}


Table 1 (continued)

\begin{tabular}{|c|c|c|c|c|c|c|c|c|c|c|c|c|c|}
\hline Stations & $\begin{array}{l}R \\
\left(\mu \mathrm{g} \mathrm{L}^{-1}\right)\end{array}$ & $\mathrm{CPI}_{15-40}$ & $\begin{array}{l}\text { Pr/ } \\
\text { Phy }\end{array}$ & $\begin{array}{l}\text { Ter/ } \\
\text { aq }\end{array}$ & MH & $\begin{array}{l}\sum_{16} \\
\text { PAHs } \\
\left(\mathrm{ng} \mathrm{L}^{-1}\right)\end{array}$ & $\begin{array}{l}\sum_{19} \\
\text { PAHs } \\
\left(\text { ng L }^{-1}\right)\end{array}$ & $\begin{array}{l}\sum_{29} \text { PAHs }_{-} \\
\mathrm{P}+\mathrm{A} \\
\left(\mathrm{ng} \mathrm{L}^{-1}\right)\end{array}$ & $\begin{array}{l}\text { LMW/ } \\
\mathrm{HMW}_{\mathrm{P}+\mathrm{A}}\end{array}$ & $\begin{array}{l}\text { Alk/ } \\
\text { Par }\end{array}$ & $\begin{array}{l}\text { Flt/ } \\
(\mathrm{Pyr}+\text { Flt })\end{array}$ & $\begin{array}{l}\mathrm{LMW}_{\mathrm{P}+\mathrm{A}} \\
(\%)\end{array}$ & $\begin{array}{l}\mathrm{HMW}_{\mathrm{P}+\mathrm{A}} \\
(\%)\end{array}$ \\
\hline S35 & 0.21 & 3.35 & 4.21 & 0.08 & $n-\mathrm{C}_{15}$ & 0.64 & 0.73 & 1.28 & 2.26 & 0.84 & $n d$ & 69.29 & 30.71 \\
\hline S36 & 0.19 & 4.00 & 3.40 & 0.08 & $n-\mathrm{C}_{15}$ & 0.89 & 1.03 & 4.01 & 0.67 & 3.86 & 0.05 & 40.08 & 59.92 \\
\hline
\end{tabular}

$n d$ not determined, bld below detection limit, $R$ total resolved $n$-alkanes from $n$ - $\mathrm{C}_{15}$ to $n$ - $\mathrm{C}_{40}$ including two isoprenoids, pristane (Pr) and phytane (Phy), Ter/aq terrigenous/aquatic ratio: $n-\mathrm{C}_{29}+n-\mathrm{C}_{31}+n-\mathrm{C}_{33} / n-\mathrm{C}_{15}+n-\mathrm{C}_{17}+n-\mathrm{C}_{19}, C P I_{15-40}$ carbon preference index: $\sum$ odd $n$-alkanes $n$ - $\mathrm{C}_{15}-n$ - $\mathrm{C}_{40} / \sum$ even $n$-alkanes $n-\mathrm{C}_{15}-n-\mathrm{C}_{40}, P r / P h y$ pristane to phytane ratio, $M H$ major hydrocarbons, $\sum_{19} P A H_{-P}$ sum of 19 parent PAHs, $\sum_{29} P A H_{-P+A}$ sum of 29 parent and alkylated PAHs, $\sum_{16} P A H$ sum of the 16 priority PAHs listed by EPA, Alk/Par sum of alkylated PAHs/sum of parent PAHs, Flt fluoranthene, Pyr pyrene, $L M W$ low molecular weight (two to three rings), $H M W$ high molecular weight ( $\geq 4$ rings)

a notable depletion of the HMW compounds in the $n-\mathrm{C}_{24}$ $n-\mathrm{C}_{40}$ range (Table 1 ; Fig. $4 \mathrm{a}$ ). AHs display monomodal distributions centred on $n-\mathrm{C}_{15}$ or $n-\mathrm{C}_{17}$, with no dominance of odd or even compounds, resulting in $\mathrm{CPI}_{15-40}$ values close to 1 (1.4 \pm 0.2$)$ (Table 1; Fig. 3b). The Pr/Phy values are very close to $1(1.1 \pm 0.2)$ (Table 1$)$, and the Ter/aq ratio is low with an average of $0.03 \pm 0.02$ (Fig. 3c).

Concentrations of $\sum_{29}$ PAHs $_{-\mathrm{P}+\mathrm{A}}$ varied from 3.7 (S14) to $12.5 \mathrm{ng} \mathrm{L}^{-1}$ (M9) (mean $7.8 \pm 3 \mathrm{ng} \mathrm{L}^{-1}$ ) (Table 1; Fig. 3d, Fig. S2a). These stations are characterized by the dominance of LMW PAH compounds over HMW compounds (LMW/ HMW $_{-\mathrm{P}+\mathrm{A}}=10 \pm 4$ ) (Table 1; Figs. $3 \mathrm{e}$ and 5a) and the dominance of alkylated homologues over parent compounds (with most of Alk/Par $>2$, mean $4.5 \pm 3.4$ ) (Fig. 3f). LMW compounds represent $90 \pm 3.4 \%$ of total $\mathrm{PAHs}$, for which alkylated phenanthrenes/anthracenes $\left(\mathrm{C}_{1^{-}}, \mathrm{C}_{2^{-}}\right.$and $\mathrm{C}_{3}$-Phe/Ant $)$ are the major compounds $(51 \pm 16 \%$ of total PAHs), followed by naphthalenes (Naph, $\mathrm{C}_{1^{-}}, \mathrm{C}_{2^{-}}, \mathrm{C}_{3^{-}} \mathrm{Naph}$ ) (Fig. 5a). HMW
PAHs are also present with very low concentrations. The Flt/ (Pyr + Flt) ratios are in the range $0.4-0.5$ at S14, M5, M6 and S19 and $<0.4$ at S15, M9-M11 and M13 (Table 1).

\section{Group $G^{\prime 2}$}

This group comprises stations M3 and M3 $3_{-\mathrm{AR}}$, from the northern coast of Sfax, and stations M4-AR, M8 and M8 $8_{-\mathrm{AR}}$, from the southern coast of Sfax (Fig. 2). These stations are distinguished by the highest $R$ concentrations, which vary from 0.1 (M3) to $3.24 \mu \mathrm{g} \mathrm{L}^{-1}$ (M8 ${ }_{-\mathrm{AR}}$ ) (mean $1.73 \pm 1.2 \mu \mathrm{g} \mathrm{L}^{-1}$ ) (Table 1; Fig. 3a, Figs. S1a and S3a). A bimodal distribution of $n$-alkanes is observed, in which LMW compounds are centred on $n-\mathrm{C}_{15}$ or $n-\mathrm{C}_{17}$ and HMWs are centred on $n-\mathrm{C}_{29}$ or $n-\mathrm{C}_{31}$ with a clear dominance of LMWs (Fig. 4b). CPI $\mathrm{C}_{15-40}$ values are close to 2 (mean of $1.86 \pm 0.33$ ), indicating a slight odd carbon-numbered predominance (Fig. 3b). The Pr/Phy a)

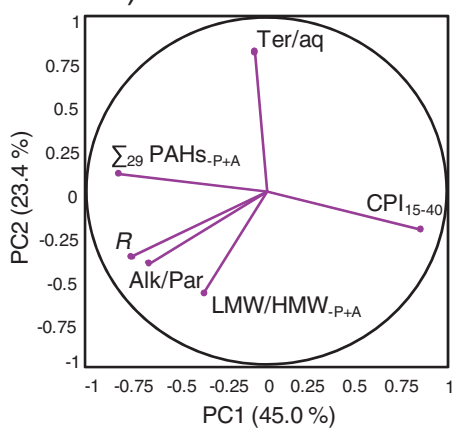

b)

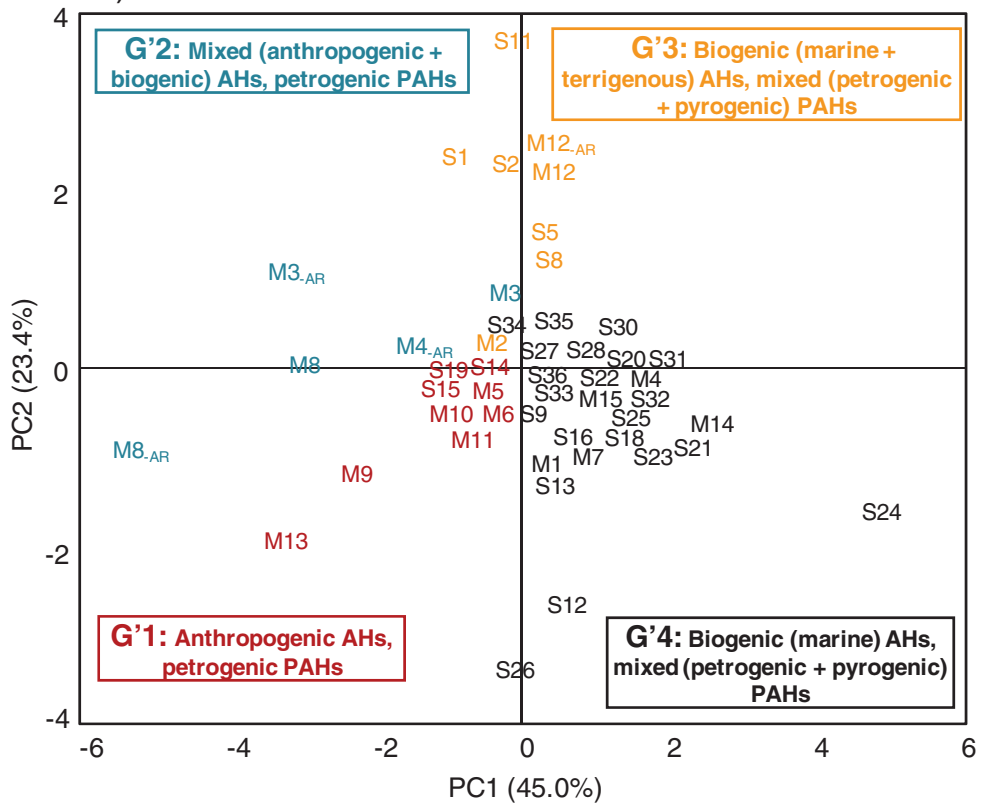

Fig. 2 Principal component analysis (PCA) of hydrocarbon dataset. a Correlation circle. b Projection of samples on the first factorial plane (PC1 versus PC2) 
a)

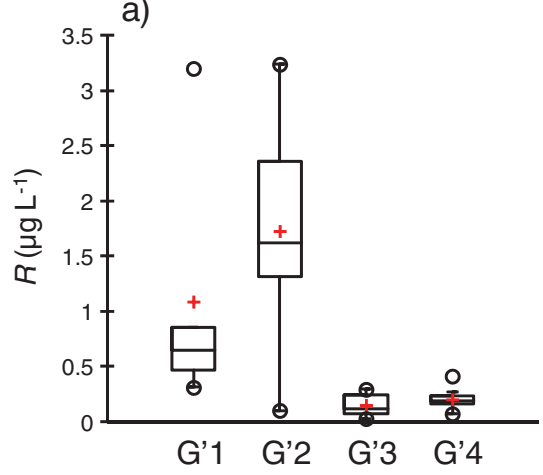

d)

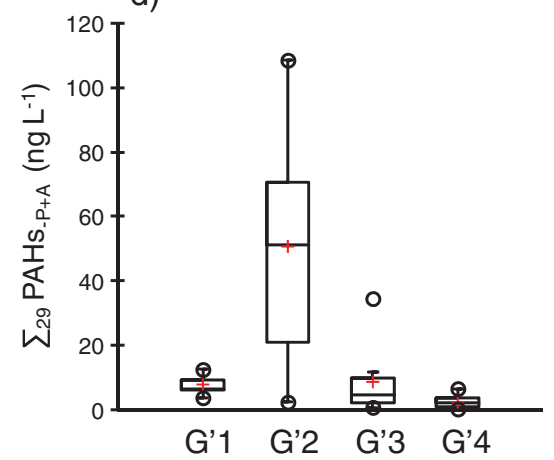

b)

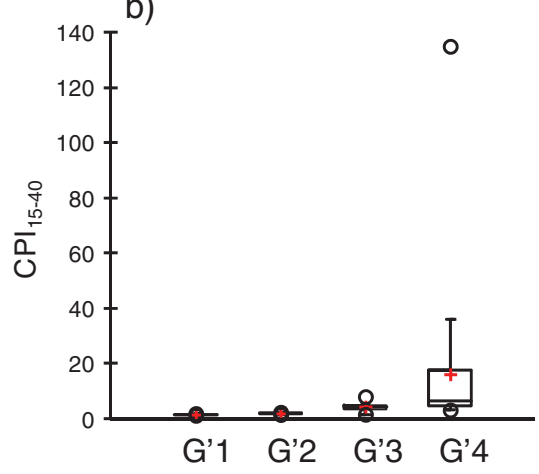

e)

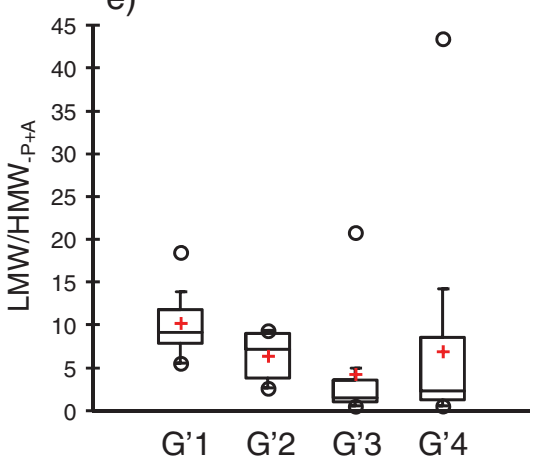

c)

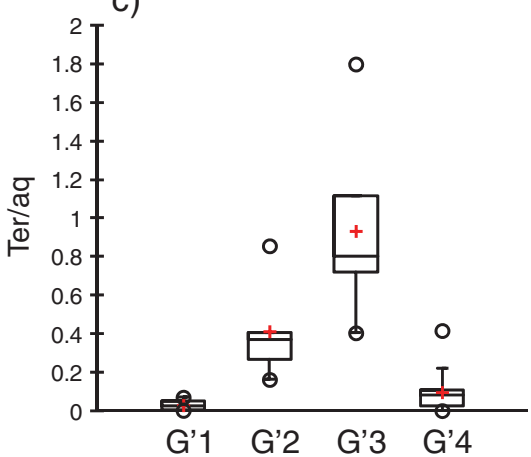

f)

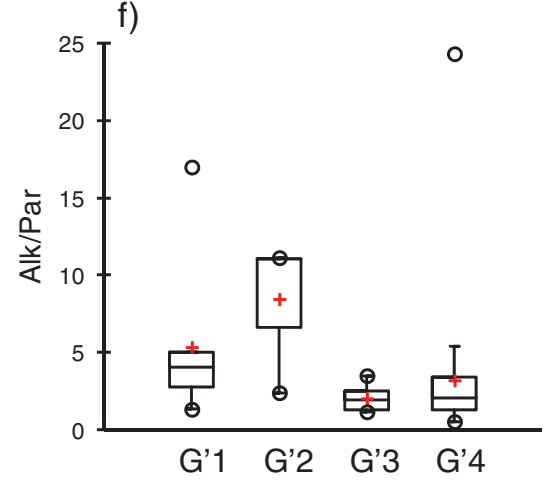

Fig. 3 Box-and-whisker plots of main hydrocarbon parameters with regard to the four clusters identified from the hierarchical ascendant classification. a Total resolved $n$-alkanes $(R)$. b CPI ${ }_{15-40}$. c Ter/aq. d $\sum_{29} \mathrm{PAH}_{-\mathrm{P}+\mathrm{A}}$. e LMW/HMW $-\mathrm{P}+\mathrm{A}$. f Alk/Par. The four clusters are as follows: G'1 (S14, S15, M5, M6, S19, M9-M11, M13), G'2 (M3, M3.

AR, M4 $\left.4_{-A R}, M 8, M 8_{-A R}\right), G^{\prime} 3$ (M2, S1, S2, S5, S8, S11, M12, M12 - AR) and G'4 (M1, S9, S12, S13, S16, S18, M4, M7, S20-S26, M14, M15, S27-S36). The black line and red cross represent the median and mean values, respectively a)

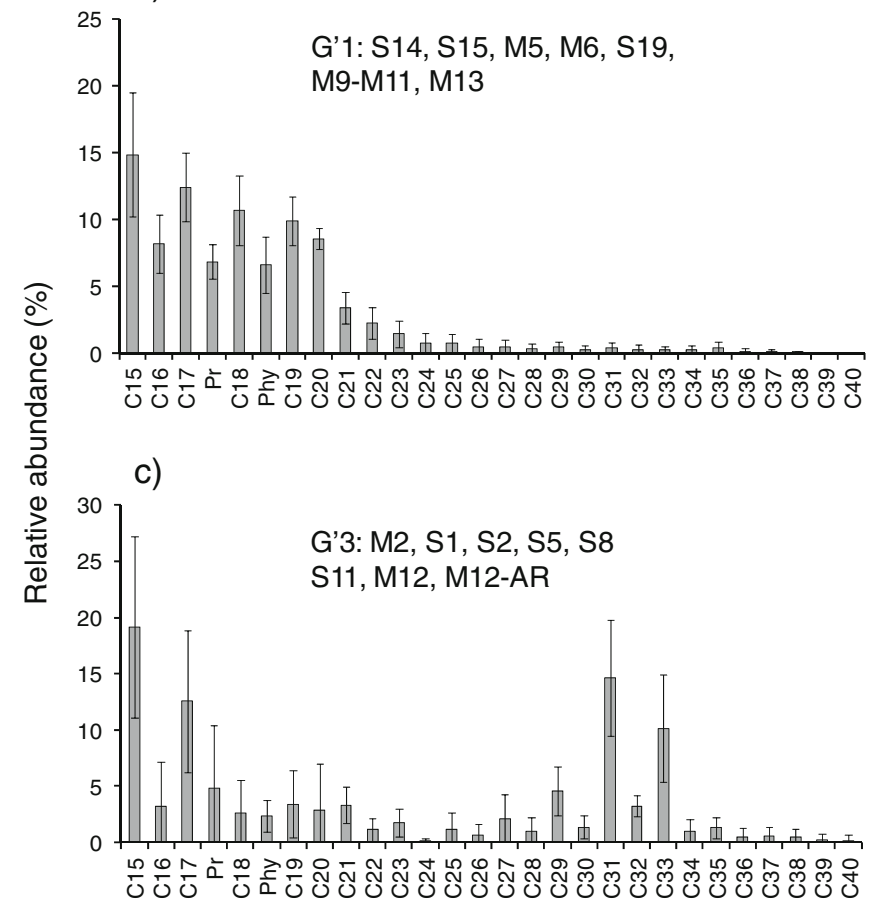

b)

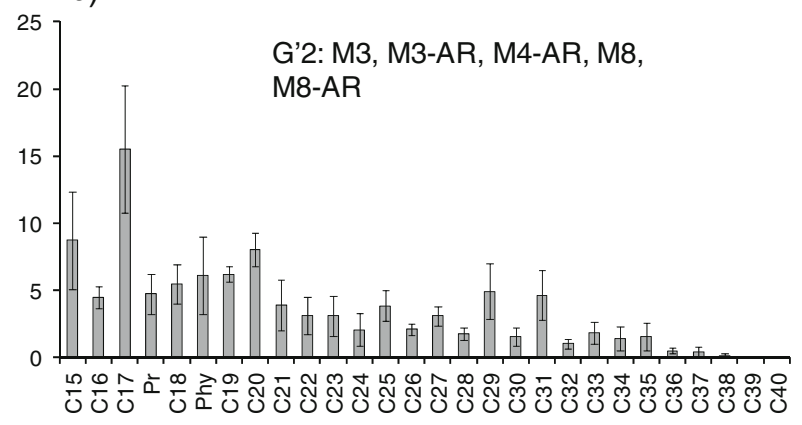

d)

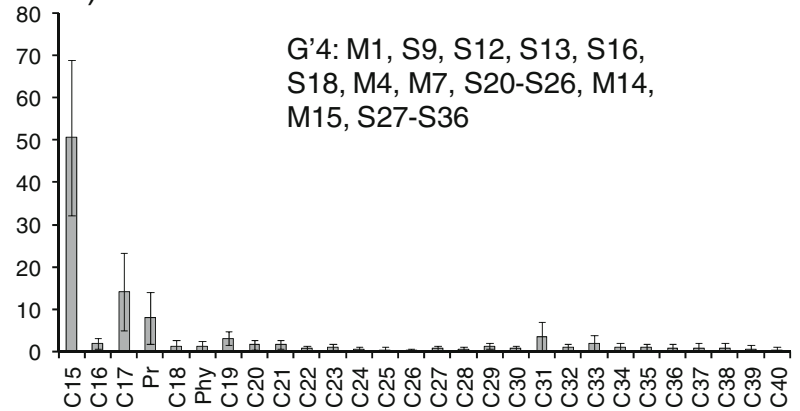

Fig. 4 Relative abundances of particulate AHs (\%) for stations a G'1 (S14, S15, M5, M6, S19, M9-M11, M13), b G'2 (M3, M3_-AR, M4_-AR, M8, M8. AR), c G'3 (M2, S1, S2, S5, S8, S11, M12, M12_AR), d G'4 (M1, S9, S12, S13, S16, S18, M4, M7, S20-S26, M14, M15, S27-S36) 


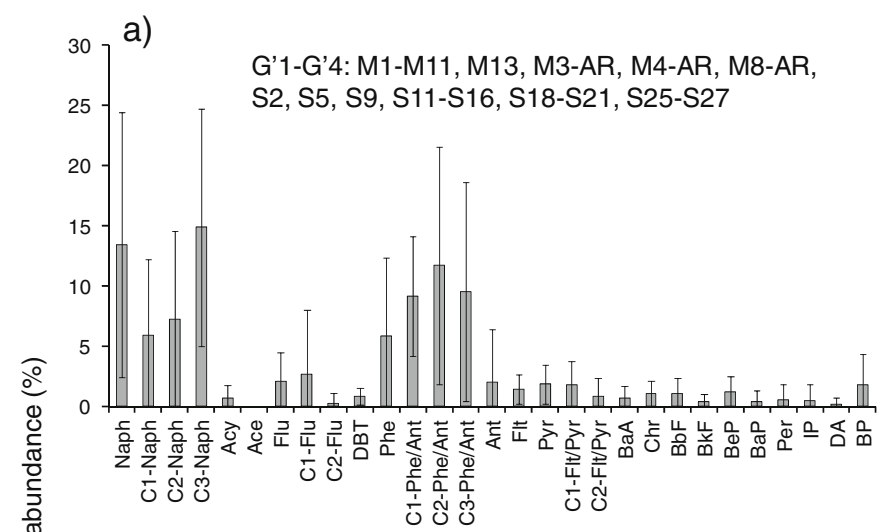

c)

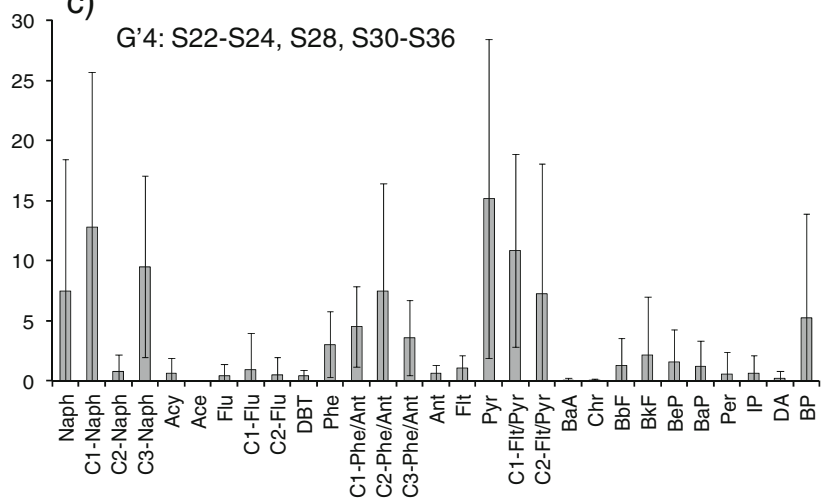

Fig. 5 Relative abundances of particulate PAHs (\%) for stations a $\mathrm{G}^{\prime} 1-\mathrm{G}$ ' 4 (M1-M11, M13, M3-AR, M4-AR, M8-AR, S2, S5, S9, S11-S16, S18-S21, S25-S27), b G'3, G'4 (S1, S8, M12, M12-AR, M14, M15), c

ratio is close to $1(0.8 \pm 0.2)$, and the Ter/aq ratio is mainly $<1$ (mean $0.41 \pm 0.26$ ) (Table 1; Fig. 3c).

G'2 exhibits the highest PAH concentrations, with $\sum_{29}$ PAHs $_{-\mathrm{P}+\mathrm{A}}$ varying from 2.3 (M3) to $108.6 \mathrm{ng} \mathrm{L}^{-1}$ (M8 $\left.8_{-\mathrm{AR}}\right)$ (mean $51 \pm 42 \mathrm{ng} \mathrm{L}^{-1}$ ) (Table 1; Fig. 3d, Figs. S2a and S3b). $\mathrm{PAH}$ molecular distributions reveal the predominance of LMWs (LMW/HMW $-\mathrm{P}+\mathrm{A}=6.4 \pm 3$; Fig. 3e), as well as the alkylated compounds, with the highest Alk/Par ratio $>2$ (mean $6.6 \pm 2.7$; Table 1; Fig. 3f). LMW PAHs represent $84 \pm 8 \%$ of total PAHs, of which naphthalenes (Naph, $\mathrm{C}_{1^{-}}, \mathrm{C}_{2^{-}}, \mathrm{C}_{3^{-}} \mathrm{Naph}$ ) and alkylated phenanthrenes/anthracenes $\left(\mathrm{C}_{1^{-}}, \mathrm{C}_{2^{-}}, \mathrm{C}_{3}-\mathrm{Phe} /\right.$ Ant) are the major compounds (Fig. 5a). Nevertheless, HMWs are also present in significant proportions and they represent $20-30 \%$ of total PAHs, at stations $\mathrm{M} 3_{-\mathrm{AR}}$ and M4 $\mathrm{AR}$, with Pyr and alkylated fluoranthenes/pyrenes $\left(\mathrm{C}_{1^{-}}, \mathrm{C}_{2}-\mathrm{Flt} /\right.$ Pyr) as major compounds (Table 1; Fig. 5a). The Flt/(Pyr + Flt) ratio is $>0.5$ at $\mathrm{M} 3$, in the range $0.4-0.5$ at $\mathrm{M} 3_{-\mathrm{AR}}, \mathrm{M} 4_{-\mathrm{AR}}$ and M8, and $<0.4$ at M8 $8_{-A R}$ (Table 1$)$.

\section{Group $G^{\prime} 3$}

Group $\mathrm{G}^{\prime} 3$ comprises station M2, from the Kerkennah Islands, $\mathrm{S} 1, \mathrm{~S} 2$, S5, S8 and S11, from the northern coast of Sfax, and M12 and M12 -AR from the Mahrès delegation (Fig. 2). $R$

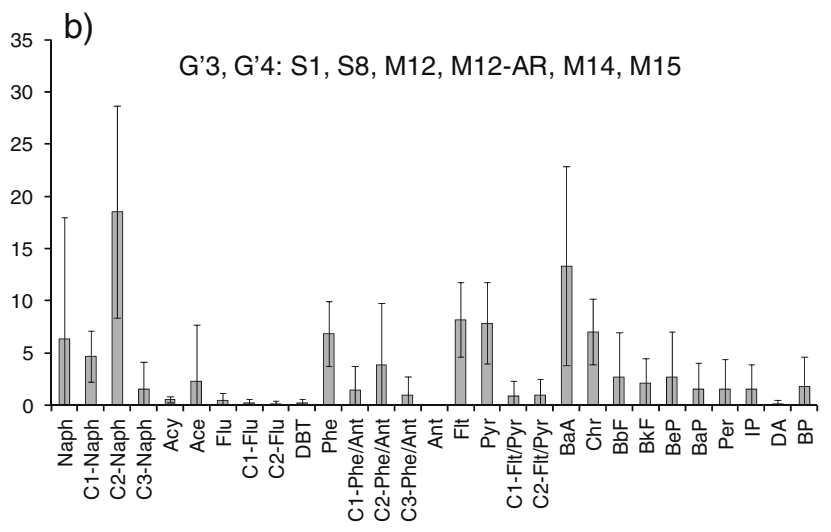

G'4 (S22-S24, S28, S30-S36). Note that station S29 is not included in figures since it displays PAH concentration below detection limit

concentrations are the lowest and range from 0.03 (M2) to $0.29 \mu \mathrm{g} \mathrm{L}^{-1}\left(\mathrm{~S} 2\right.$ and $\mathrm{M} 12_{\text {-AR }}$ ) (mean $0.15 \pm 0.10 \mu \mathrm{g} \mathrm{L}^{-1}$ ) (Table 1; Fig. 3a, Figs. S1a and S3a). All stations display bimodal distributions in which light compounds are centred on $n-\mathrm{C}_{15}$ or $n-\mathrm{C}_{17}$ and heavy ones on $n-\mathrm{C}_{31}$ or $n-\mathrm{C}_{33}$ (Fig. $4 \mathrm{c}$ ). The AH molecular profiles reveal a large odd-to-even carbonnumbered predominance that results in $\mathrm{CPI}_{15-40}$ values widely $>2$ (mean $4.2 \pm 1.9$ ) (Table 1; Fig. 3b). The $\mathrm{Pr} / \mathrm{Phy}$ ratio is $>1$ at M2, S2 and S11 stations (Table 1), and the Ter/aq ratio is the highest of all groups with values ranging from 0.4 (S8) to 1.8 (S11) (mean $0.9 \pm 0.4$; Table 1; Fig. 3c).

$\mathrm{G}^{\prime} 3$ records moderate PAH concentrations, with $\sum_{29}$ PAHs. $\mathrm{P}+\mathrm{A}$ varying from 0.7 (M12) to $34.5 \mathrm{ng} \mathrm{L}^{-1}$ (S1) (mean $7.6 \pm 11.5 \mathrm{ng} \mathrm{L}^{-1}$ ) (Table 1; Fig. 3d, Figs. S2a and S3b). This group of stations present the lowest alkylated homologue contributions with a mean Alk/Par ratio of $2.0 \pm 0.8$ (Figs. $3 \mathrm{f}$ and $5 \mathrm{~b}$ ). While the molecular distribution reveals the dominance of naphthalenes as major LMW compounds, especially at M2, S2, S5 and S11 (Figs. 3e and 5a), an equal contribution between LMW and HMW compounds is observed for stations S1, S8, M12 and M12 ${ }_{-A R}$, with HMW PAHs representing $54.5 \pm 8.2 \%$ of total PAHs (Figs. 3 e and 5 b). The Flt/(Pyr + Flt) ratio is in the range $0.4-0.5$ at all stations, except for M2, S5 and M12 with values $>0.5$ (Table 1$)$. 
a)

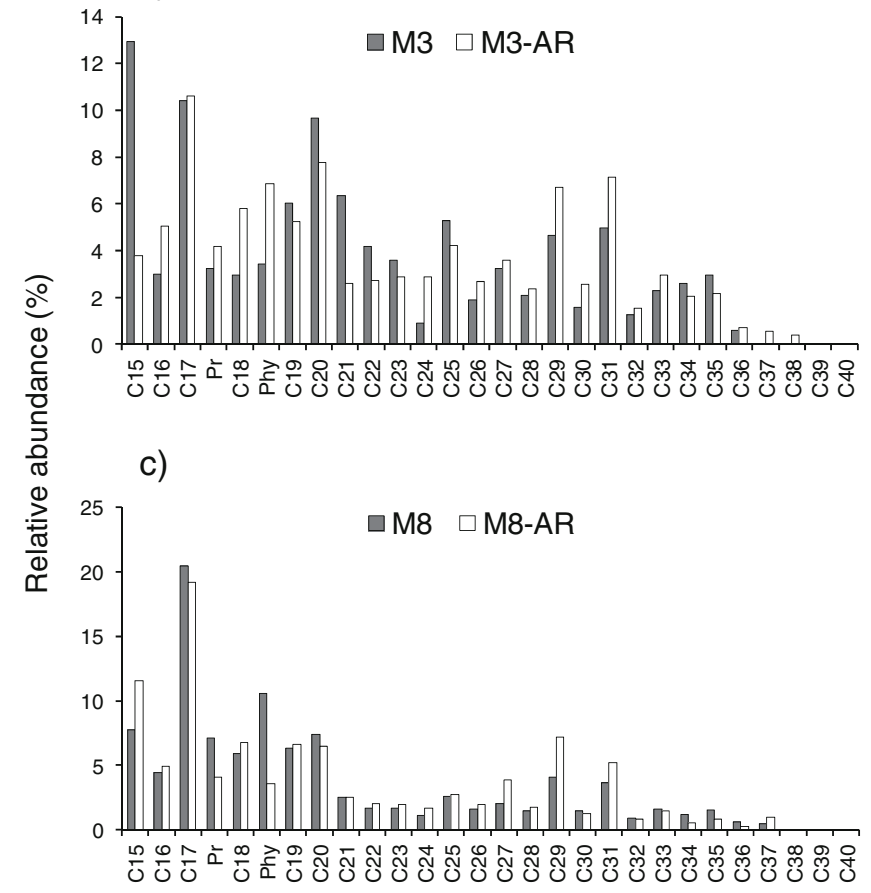

b)

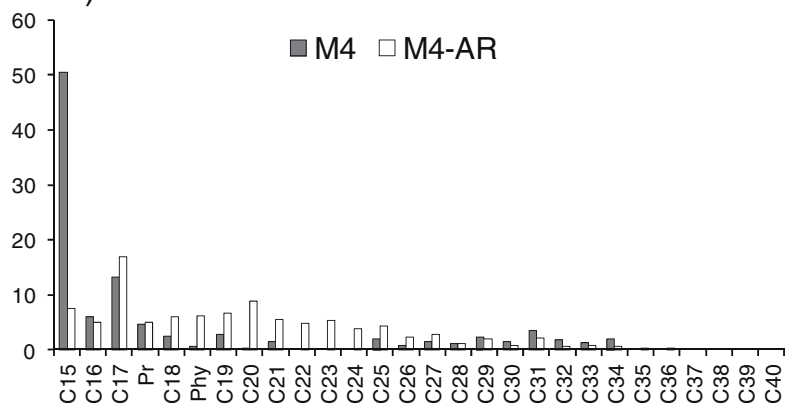

d)

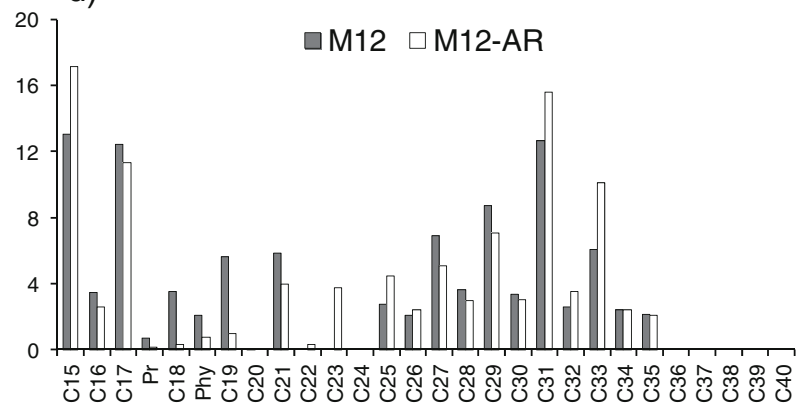

Fig. 6 Relative abundances of particulate AHs (\%) of stations collected before and after rainfall events. a M3 and M3 M8 - AR. d M12 and M12-AR

\section{Group $G^{\prime} 4$}

The G'4 group contains stations M1, from the Kerkennah Islands; S9, S12 and S13, from the northern coast of Sfax; S16, S18, M4, M7 and S20-S26, from the southern coast of Sfax; M14 and M15, from the Mahrès delegation and all stations from the Gabès-Ghannouch coastal area (S27-S36) (Fig. 2). $R$ concentrations are low to moderate and range from 0.07 (M4) to $0.36 \mu \mathrm{g} \mathrm{L}^{-1}$ (S23) (mean $0.20 \pm 0.07 \mu \mathrm{g} \mathrm{L}{ }^{-1}$ ) (Table 1; Fig. 3a, Fig. S1a). G'4 molecular distributions reveal a monomodal distribution with $n-\mathrm{C}_{15}$ as a major compound, which reaches up to $82 \%$ of total AHs at M14 (mean $52 \pm 18 \%$; Fig. 4d). The $\mathrm{CPI}_{15-40}$ values are $>3$ at all stations (mean $16 \pm 26$ ), highlighting a large odd carbon-numbered compound predominance (Fig. 3b). This group is also characterized by a high Pr/Phy contribution with most of the values $>1$ (Table 1). G'4 is also marked by the highest LMW contributions $\left(n-\mathrm{C}_{15}\right.$ and $n-\mathrm{C}_{17}$ representing $65 \pm 16 \%$ of the total $R$ concentration), resulting in low Ter/aq values (mean $0.097 \pm 0.094$ ) (Fig. 3c).

PAH concentrations are the lowest with $\sum_{29}$ PAHs $_{-\mathrm{P}+\mathrm{A}}$ ranging from $b d l(\mathrm{~S} 29)$ to $6.5 \mathrm{ng} \mathrm{L}^{-1}$ (S34) (mean $2.4 \pm 1.7 \mathrm{ng} \mathrm{L}^{-1}$ ) (Table 1; Fig. 3d, Fig. S2a). Stations S22S24, M14, M15, S28 and S30-S36 display molecular profiles with a moderate-to-high contribution of HMW compounds, which represent $47 \pm 13 \%$ of total PAHs. Among these HMW PAHs, Pyr and alkylated fluoranthenes/pyrenes $\left(\mathrm{C}_{1}-\right.$ and $\mathrm{C}_{2}-$ Flt/Pyr), BaA and BP are the major compounds (Fig. 5b, c).
These stations display moderate alkylated homologue contributions with Alk/Par values 2 (mean $2.5 \pm 1.3$ ) (Table 1; Fig. $5 \mathrm{~b}, \mathrm{c})$. For the rest of the stations, $\mathrm{PAHs}_{-\mathrm{P}+\mathrm{A}}$ distribution patterns reveal the dominance of LMW compounds $(87 \pm 10 \%$; mean LMW/HMW $-\mathrm{P}+\mathrm{A}$ ratio of $12.6 \pm 11.9)$, in which naphthalenes (Naph, $\mathrm{C}_{1^{-}}, \mathrm{C}_{2^{-}}, \mathrm{C}_{3^{-}} \mathrm{Naph}$ ), Phe and/or alkylated phenanthrenes/anthracenes $\left(\mathrm{C}_{1^{-}}, \mathrm{C}_{2^{-}}, \mathrm{C}_{3^{-}}\right.$-Phe/Ant) are the major compounds (Figs. 3e and 5a). For these stations, the alkylated homologues were relatively abundant, with Alk/ Par values $>2$ (mean $4.1 \pm 5.9$ ). The Flt $/(\mathrm{Pyr}+\mathrm{Flt}$ ) ratio is $>0.5$ at M1, S9, M14 and M15, in the range of 0.4-0.5 at S12, S18 and M4, and <0.4 at S20-S25 and S30-S36 (Table 1).

\section{Hydrocarbon distributions after rainfall}

$R$ concentrations of samples collected after rainfall (M3-AR, $\mathrm{M} 4_{-\mathrm{AR}}, \mathrm{M} 8_{-\mathrm{AR}}$ and $\mathrm{M} 12_{-\mathrm{AR}}$ ), which ranged from 0.29 to $3.24 \mu \mathrm{g} \mathrm{L}^{-1}$, increased by a factor of 2-23.5 compared to those of samples collected before rain at the same sites (Table 1). This concentration enhancement is associated with a decrease (M3 $3_{-A R}$ and M4-AR $)$ or an increase (M8 $8_{-A R}$ and

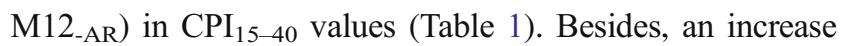
in Ter/aq ratio is recorded at all stations, particularly at M3. AR and M12 $2_{-A R}$ (Table 1; Fig. 6a-d).

$\sum_{29}$ PAHs $_{\text {P }+ \text { A }}$ concentrations ranged between 2.10 and $108.6 \mathrm{ng} \mathrm{L}^{-1}$ in samples collected after rain, underlying an increase by a factor of 1.5-22 compared to those collected before rain at the same sites (Table 1). These after-rain 
a)

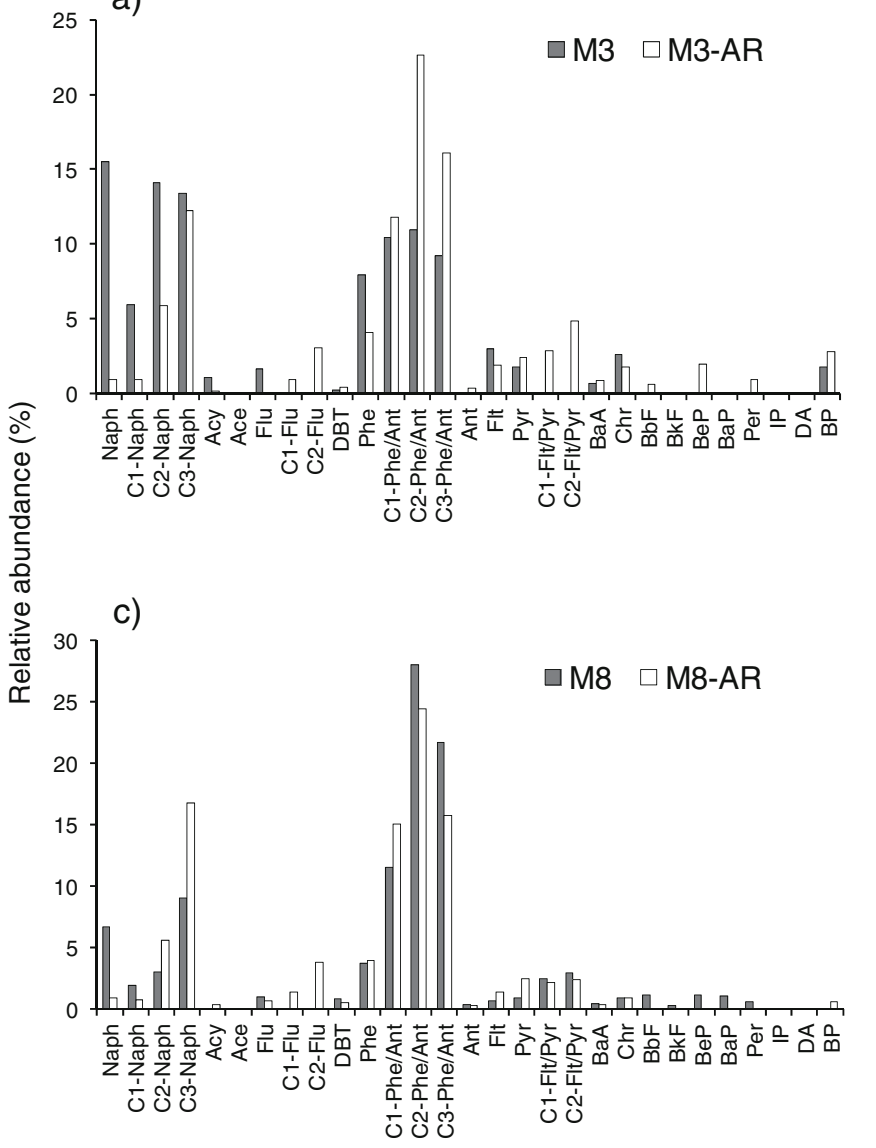

b)

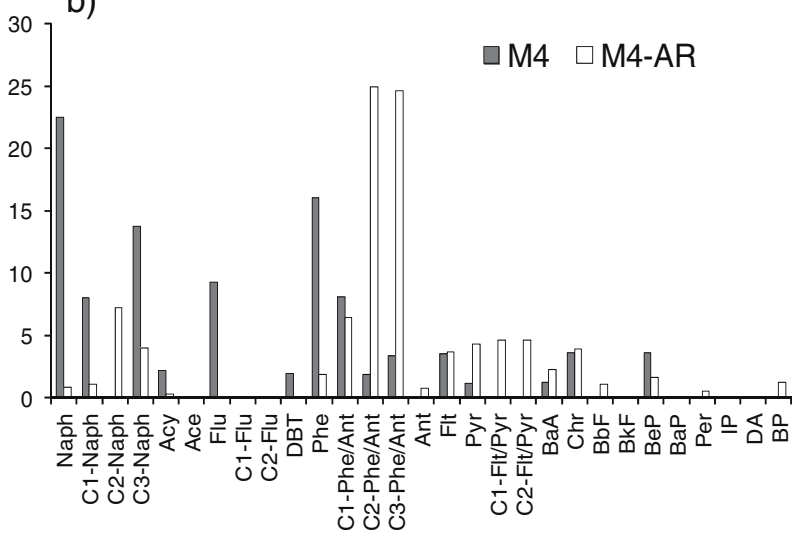

d)

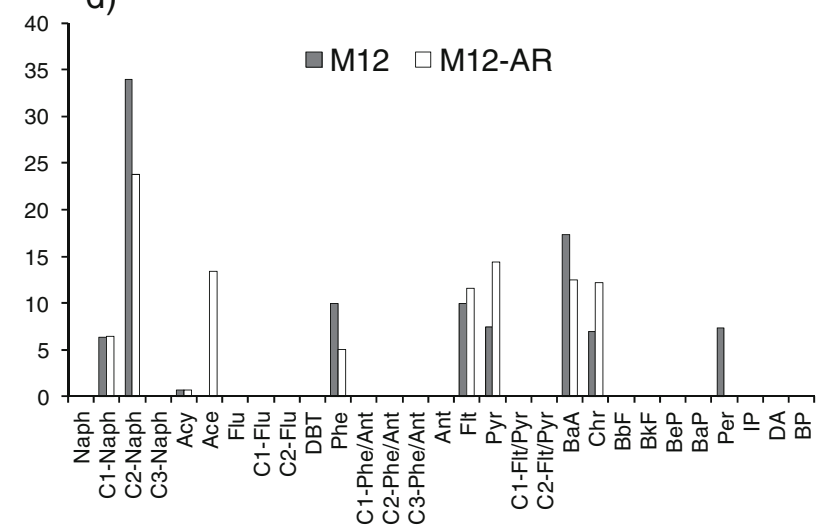

Fig. 7 Relative abundances of particulate PAHs (\%) of stations collected before and after rainfall events. a M3 and M3 $-\mathrm{AR}$. b M4 and M4-AR. c M8 and M8_-AR. d M12 and M12-AR

samples display thus the highest $\mathrm{PAH}_{-\mathrm{P}+\mathrm{A}}$ concentrations recorded during this study. This concentration increase is associated with a decrease (M3 ${ }_{-\mathrm{AR}}$ and $\left.\mathrm{M} 4_{-\mathrm{AR}}\right)$, an increase (M8

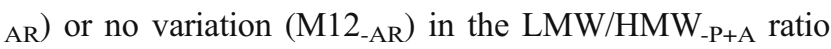
(Table 1; Fig. 7a-d); Alk/Par ratios strongly increase for M3. AR, M4-AR and M8-AR but decrease for M12 ${ }_{-A R}$, while Flt/ $\left(\right.$ Flt + Pyr) ratios slightly decrease for $\mathrm{M} 3_{-\mathrm{AR}}, \mathrm{M} 4_{-\mathrm{AR}}, \mathrm{M} 8_{-\mathrm{AR}}$ and M12-AR (Table 1).

\section{Correlation between particulate hydrocarbons and biogeochemical parameters}

Table 2 displays Spearman's rank-order correlation matrix for particulate hydrocarbons and biogeochemical parameters. Note that the spatial distribution of biogeochemical parameters is provided in Fig. S4 and fully described in Fourati et al. (2017). $R$ concentration is significantly positively correlated with $\sum_{29} \mathrm{PAHs}_{-\mathrm{P}+\mathrm{A}}$ concentration ( $r=0.65, n=48, p<0.05)$, suggesting that AHs and PAHs in this coastal area might have common origins or undergo similar processes. Evidence of this coupling can also be seen from PCA, in which $\mathrm{AH}$ and PAH concentrations are located on principal component 1 (PC1)
(Fig. 2). Correlations between $\sum_{29} \mathrm{PAHs}_{-\mathrm{P}+\mathrm{A}}$ and POC and PON concentrations $(r=0.56-0.60, n=23$, $p<0.05)$ also suggest a possible coupling between PAHs and organic carbon in the particulate phase. On the other hand, no significant relation is observed between AH content, including $R, n-\mathrm{C}_{15}$ and $n-\mathrm{C}_{17}$, and TChl $a$ concentration, neither when considering all stations ( $r=-0.08-0.21, n=23, p>0.05$ ), nor when taking into consideration individual groups of stations (data not shown). LMW PAHs $-\mathrm{P}+\mathrm{A}$ are found to correlate with $n$ $\mathrm{C}_{17}$ considering all stations $(r=0.67, n=23, p<0.05)$ and considering groups $G^{\prime} 1, G^{\prime} 2$ and $G^{\prime} 4$ (data not shown). As reported by Fourati et al. (2017), the correlations between biogeochemical parameters point out the strong interconnections between phytoplankton biomass, nutrients and organic carbon pool in this area. Particulate OM content recorded in surface waters may be derived from autochthonous biological activity, terrestrial material entering marine waters via channels, wadis and runoffs, as suggested by the positive significant correlation between particulate matter and $\mathrm{Si}(\mathrm{OH})_{4}(r=0.44-0.68, n=23$, $p<0.05$ ), or sediment particles, which are enriched in macrophyte material in decomposition. 


\section{Discussion}

Significance of particulate AH and PAH concentrations

Studies dealing with hydrocarbons in marine waters have mostly focused on PAHs. That is why our AH concentrations cannot be compared with as many works as for PAHs. Particulate total $R$ concentrations $\left(n-\mathrm{C}_{15}-n-\mathrm{C}_{40}\right.$ with $\mathrm{Pr}$ and Phy) recorded here in the surface coastal waters of the Gulf of Gabès ( 0.03 to $\left.3.24 \mu \mathrm{g} \mathrm{L}^{-1}\right)$ are within the same range as those observed in the Marseilles coastal area $\left(\sum n-\mathrm{C}_{16}-n-\mathrm{C}_{35}\right.$ $0.04-4.30 \mu \mathrm{g} \mathrm{L}^{-1}$; Guigue et al. 2011) and the Tyrrhenian Sea ( $\sum n-\mathrm{C}_{14}-n-\mathrm{C}_{34}$ 0.27-5.62 $\mu \mathrm{g} \mathrm{L}{ }^{-1}$; Cincinelli et al. 2001). On the other hand, our measured concentrations are much higher than those measured in the northern Black Sea $\left(\sum n-\mathrm{C}_{14}-n-\mathrm{C}_{36}\right.$ 0.002-0.2 $\mu \mathrm{g} \mathrm{L}^{-1}$; Maldonado et al. 1999) and in the Catalan Sea (northwestern Mediterranean Sea) $\left(\sum n-\mathrm{C}_{15}-n-\mathrm{C}_{35} 0.01-\right.$ $0.02 \mu \mathrm{g} \mathrm{L}^{-1}$; Dachs et al. 1999) (Table 3).

Particulate PAH concentrations measured here in the surface waters of the Gulf of Gabès are five times higher when alkylated homologues are included $\left(\sum_{29}\right.$ PAHs $_{-\mathrm{P}+\mathrm{A}} b d l-$ $\left.108.6 \mathrm{ng} \mathrm{L}^{-1}\right)$ than when only parent compounds are considered $\left(\sum_{19}\right.$ PAHs $\left._{-\mathrm{P}} b d l-26 \mathrm{ng} \mathrm{L}^{-1}\right)$. Considering $\sum_{19}$ PAHs$_{-\mathrm{P}}$, concentrations are within the range as those reported in the Marseilles coastal area $\left(\sum_{17}\right.$ PAHs $_{-P} 1.9-21 \mathrm{ng} \mathrm{L}^{-1}$; Guigue et al. 2011) and in the Rhône River Delta ( $\sum_{11}$ PAHs $_{-P} 1.1-$ $19 \mathrm{ng} \mathrm{L}^{-1}$; Bouloubassi and Saliot 1991). However, they are higher compared to those reported in the Black Sea $\left(\sum_{15}\right.$ PAHs $_{\text {P }}$ 0.05-0.27 ng L ${ }^{-1}$; Maldonado et al. 1999) and in the Baltic Sea $\left(\sum_{15}\right.$ PAHs $_{-\mathrm{P}} 1.8-8.1 \mathrm{ng} \mathrm{L}^{-1} ; \sum_{11}$ PAHs $_{-\mathrm{P}} 0.05-$ $0.33 \mathrm{ng} \mathrm{L}^{-1}$; Broman et al. 1991; Witt 2002). Cincinelli et al. (2001), El Nemr and Abd-Allah (2003) and Wu et al. (2011) reported higher concentrations in the Tyrrhenian Sea $\left(\sum_{15}\right.$ PAHs $_{\text {-P }}$ 550-7540 ng L ${ }^{-1}$ ), in the Egyptian coastal waters ( $\sum_{7}$ PAHs $_{-\mathrm{P}}$ 13-120 ng L ${ }^{-1}$ ) and in the South China Sea ( $\sum_{15}$ PAHs $_{-P} 10.3-45.5 \mathrm{ng} \mathrm{L}^{-1}$ ), respectively (Table 3 ). On the other hand, our $\sum_{29}$ PAHs $_{-\mathrm{P}+\mathrm{A}}$ concentrations are higher than those measured in the Gulf of Fos-sur-Mer $\left(\sum_{\sim} 80\right.$ PAHs $\mathrm{P}+\mathrm{A} 2-37 \mathrm{ng} \mathrm{L}^{-1}$; Bouloubassi and Saliot 1991) and in the Gulf of Mexico ( $\sum_{43}$ PAHs $_{-\mathrm{P}+\mathrm{A}}$ 0.30-1.3 ng L ${ }^{-1}$; Adhikari et al. 2015), but lower than those recorded in the Marseilles coastal area $\left(\sum_{32}\right.$ PAHs $_{-\mathrm{P}+\mathrm{A}}$ 11-208 ng L ${ }^{-1}$; Guigue et al. 2011) (Table 3).

\section{Origin and potential sources of particulate hydrocarbons}

G'1 hydrocarbon content $\left(\mathrm{CPI}_{15-40}, \mathrm{LMW} / \mathrm{HMW}_{-\mathrm{P}+\mathrm{A}}\right.$ and Alk/Par) reveals a strong anthropization attributed to recent/ chronic unburned oil products (Figs. 2, 3b, e, f, 4a and 5a) (Guigue et al. 2011; Mille et al. 2006; Youngblood and Blumer 1975). Therefore, it seems not relevant to use biogenic indicators and ratios (Pr/Phy and Ter/aq) for this group. Additionally, the Flt/(Pyr + Flt) ratio suggests a contribution 
Table 3 Comparison of particulate hydrocarbon concentrations recorded in the surface waters of the Gulf of Gabès with those reported in other environments (without considering the surface microlayer)

\begin{tabular}{|c|c|c|c|}
\hline References & Area & Particulate AHs $\left(\mu \mathrm{g} \mathrm{L}^{-1}\right)$ & Particulate PAHs (ng L ${ }^{-1}$ ) \\
\hline This study & SE Mediterranean Sea (Gulf of Gabès) & $\sum \mathrm{C}_{15}-\mathrm{C}_{40} 0.03-3.24$ & $\begin{array}{l}\sum_{29} \text { PAHs }_{-\mathrm{P}+\mathrm{A}} b d l-109 \\
\sum_{19} \text { PAHs }_{-\mathrm{P}} 0-26\end{array}$ \\
\hline Adhikari et al. (2015) & Gulf of Mexico & nd & $\sum_{43}$ PAHs $_{-\mathrm{P}+\mathrm{A}} 0.30-1.31$ \\
\hline Guigue et al. (2011) & NW Mediterranean Sea (Marseilles) & $\sum \mathrm{C}_{16}-\mathrm{C}_{35} 0.04-4.30$ & $\begin{array}{l}\sum_{32} \text { PAHs }_{-\mathrm{P}+\mathrm{A}} 11-208 \\
\sum_{17} \text { PAHs-P }_{1.9-21}\end{array}$ \\
\hline Wu et al. (2011) & Western Taiwan Strait. South China Sea & $n d$ & $\sum_{15}$ PAHs $_{-\mathrm{P}} 10.3-45.5$ \\
\hline El Nemr and Abd-Allah (2003) & Alexandria coast. Egypt & $n d$ & $\sum_{7}$ PAHs $_{-\mathrm{P}} 13-120$ \\
\hline Witt (2002) & Baltic Sea & nd & $\sum_{15}$ PAHs $_{-\mathrm{P}} 1.8-8.1^{\mathrm{a}}$ \\
\hline Cincinelli et al. (2001) & Tyrrhenian Sea & $\sum \mathrm{C}_{14}-\mathrm{C}_{34} 0.27-5.62$ & $\sum_{15}$ PAHs $_{-\mathrm{P}} 550-7540$ \\
\hline Dachs et al. (1999) & NW Mediterranean (Catalan Sea) & $\sum \mathrm{C}_{15}-\mathrm{C}_{35} 0.01-0.02$ & $N d$ \\
\hline Maldonado et al. (1999) & NW Black Sea shelf and slope & $\sum \mathrm{C}_{14}-\mathrm{C}_{36} 0.002-0.2$ & $\sum_{15}$ PAHs $_{-\mathrm{P}} 0.05-0.27$ \\
\hline Bouloubassi and Saliot (1991) & NW Mediterranean Sea (Gulf of Fos) & $n d$ & $\begin{array}{l}\sum_{11} \text { PAHs }_{-\mathrm{P}} 1.1-19 \\
\sum_{80} \text { PAHs }_{-\mathrm{P}+\mathrm{A}} 2-37\end{array}$ \\
\hline Broman et al. (1991) & Central Baltic Sea & nd & $\sum_{11}$ PAHs $_{-\mathrm{P}} 0.05-0.33$ \\
\hline
\end{tabular}

nd not determined

${ }^{a} \mathrm{PAH}$ concentration includes dissolved and particulate phases

of incomplete petroleum combustion residues at stations S14, M5, M6 and S19 and supports a petrogenic signature at stations S15, M9-M11 and M13 (Table 1) (Asia et al. 2009; Budzinski et al. 1997; Li et al. 2015; Yunker et al. 2002). $\mathrm{AH}$ profiles exhibit a molecular distribution similar to that recorded for mid-range distilled petroleum, particularly diesel products (Wang and Fingas 2003), very likely related to intense maritime activities.

G'2 reveals more pronounced anthropogenic inputs (hydrocarbon concentrations, Alk/Par) compared to G'1 (Figs. 2 and 3a, d, f), but also highlights, via $\mathrm{CPI}_{15-40}$ values, a slight emergence of biogenic signatures (Table 1; Fig. 3b). The latter are of marine origin, i.e. phytoplankton and macroalgae, with the presence of $n-\mathrm{C}_{15}$ and $n-\mathrm{C}_{17}$ (Blumer et al. 1971; Colombo et al. 1989; Commendatore and Esteves 2004; Goutx and Saliot 1980), and of terrestrial origin, i.e. epicuticular waxes of higher plants released from channels and wadis (especially the Sidi Salem channel), with a slight emergence of $n-\mathrm{C}_{29}$ and $n-\mathrm{C}_{31}$, as seen from Fig. $4 \mathrm{~b}$ and Ter/aq ratio (Fig. $3 \mathrm{c}$ ) (Douglas and Eglinton 1966). The Flt/(Pyr + Flt) ratio indicates the presence of pyrogenic inputs (wood, coal, grass) at M3 and petroleum-combusted PAHs at M3 $3_{-A R}, M 4_{-A R}$ and M8, and supports petrogenic signatures for M8-AR. These widespread petrogenic signatures observed in $\mathrm{G}^{\prime} 1$ and $\mathrm{G}^{\prime} 2$, which encompass the most coastal stations, are likely issued from maritime traffic (fishing and commercial harbours), especially for the southern coast of Sfax, and industrial and domestic discharges from $\mathrm{PK}_{4}$ channel (M3 and M3-AR), Sidi Salem channel (M8 and $M 8_{-A R}$ ) and El Maou wadi, which cross over several industrial areas of Sfax city and thus collect their effluents and wastes.
$\mathrm{G}^{\prime} 3$ and $\mathrm{G}^{\prime} 4$ stations have a less pronounced anthropogenic fingerprint. G'3 is characterized by the dominance of biogenic markers, that is $\mathrm{CPI}_{15-40}$ and Ter/aq (Figs. 2 and $3 b, c)$, which may be related to marine primary production, with the emergence of $n-\mathrm{C}_{15}, n-\mathrm{C}_{17}$ and $\mathrm{Pr}$, and to terrestrial inputs, with the emergence of $n-\mathrm{C}_{31}$ and $n$ $\mathrm{C}_{33}$ (Fig. 4c). For $\mathrm{G}^{\prime} 4$, the $\mathrm{AH}$ patterns reveal a typical biogenic marine contribution from phytoplankton and algae, underlying a strong primary production as observed by high $n-\mathrm{C}_{15}, n-\mathrm{C}_{17}, \mathrm{CPI}_{15-40}$ and $\mathrm{Pr} / \mathrm{Phy}$, and low Ter/aq values (Figs. 2, 3b, c and $4 d$ ). Since microalgae and macroalgae are abundant in the Gulf of Gabès (Bel Hassen et al. 2008; Drira et al. 2014; Rekik et al. 2014), this assumption seems plausible. Generally, low $\mathrm{AH}$ concentrations and the dominance of $n-\mathrm{C}_{15}$ and $n-\mathrm{C}_{17}$ characterize biogenic sources (Dauner et al. 2015). Despite this pronounced biogenic fingerprint and the high TChl $a$ concentration, no correlation was observed between $R$, $n-\mathrm{C}_{15}, n-\mathrm{C}_{17}$ and TChl a concentrations (Table 2). Therefore, a high phytoplankton biomass does not systematically induce a coupling between AH and TChl $a$ concentrations, as pointed out by Goutx and Saliot (1980).

G'3 stations from the northern coast of Sfax are marked by mixed PAH inputs for the most coastal stations connected directly to the Ezzit wadi (S1) and the $\mathrm{PK}_{4}$ outfall channel (S8). The Flt/(Flt + Pyr) values highlight PAH inputs from petroleum combustion at $\mathrm{S} 1$ and S8. Indeed, the area around the Ezzit wadi is marked by the highest SPM, POC and PON concentrations (Fig. S4). In general, HMW PAHs are preferentially adsorbed onto particulate 
OM (Gustafsson et al. 2001; Kennish 1992). This can explain the high HMW PAH contribution and the pyrogenic signature recorded at these stations (Fig. 5b). This obvious pyrogenic signature is not observed in the rest of the stations from the Sfax northern coast, where a petrogenic signature predominates with only the Flt/ $(\mathrm{Flt}+\mathrm{Pyr})$ ratio suggesting a pyrogenic contribution from wood, grass and coal (Table 1). This may be related to a lower particulate OM content (Fig. S4). Coastal stations from the Mahrès area (M12 and M12 $\left.2_{-\mathrm{AR}}\right)$ are also marked by mixed PAH sources (Fig. 5b), which could be due to inputs from natural gas burning from the British Gas plant (Fig. 1), and thus to inputs from water-air exchange. Interestingly, in $\mathrm{G}^{\prime} 3$, the Alk/Par ratio is low for all stations (Fig. 3f), which would confirm the dominance of the pyrogenic source for stations displaying mixed PAH signatures and reflect degradation processes for stations with dominant petrogenic fingerprints.

For G'4, stations M14 and M15 from the Mahrès delegation show a mixed PAH contribution, similar to that observed at M12 and M12 ${ }_{-\mathrm{AR}}$, confirming the mixed character of PAHs in this area (Fig. 5b). In the same way, El Maou wadi stations (S22-S24) in the Sfax southern coast, as well as Gabès stations (S28, S30-S36) are also marked by petrogenic and pyrogenic sources (Figs. 3e, f and 5c). For the rest of the stations (M1, S9, S12, S13, S16, S18, M4, M7, S20, S21, S25-S27), $\mathrm{PAH}$ molecular distributions reveal petrogenic signatures (LMW/HMW $-\mathrm{P}+\mathrm{A}$, Alk/Par; Figs. 3e, f and 5a). The Flt/ $(\mathrm{Flt}+\mathrm{Pyr})$ ratio highlights a supplementary pyrogenic origin from combustion of wood, grass and coal at M1 and S9 and petroleum combustion at S12, S18 and M4, and supports a $\mathrm{PAH}$ petrogenic origin at S20-S21 and S25 (Table 1).

In the Sfax coastal area, the pyrogenic PAH signature could be related to inputs from the Ezzit wadi and $\mathrm{PK}_{4}$ channel for the northern part $\left(\mathrm{G}^{\prime} 3\right)$ and the El Maou wadi for the southern part $\left(\mathrm{G}^{\prime} 4\right)$, which are known to carry numerous industrial and domestic wastes. For the Mahrès delegation ( $\left.\mathrm{G}^{\prime} 3, \mathrm{G}^{\prime} 4\right)$, the pyrogenic contribution would be mainly from atmospheric inputs since there are neither effluents nor wadis close to this zone. The widespread pyrogenic contributions observed for the rest of stations could be related to inputs from waste incinerations and motor vehicles. For the Gabès coastal area $\left(\mathrm{G}^{\prime}\right.$ 4), pyrogenic PAHs are observed for the coastal stations but also for stations off the coast. Moreover, we observe at these Gabès stations the highest $\mathrm{Si}(\mathrm{OH})_{4}$ concentrations, high POC and SPM concentrations (Fig. S4) and a positive significant correlation between $\mathrm{Si}(\mathrm{OH})_{4}$ and HMW PAH concentrations (not shown). This underlines different sources of PAHs compared to the Sfax zone, possibly with inputs from the petrochemical infrastructure and thermal power generation, as well as terrestrial inputs from wadis and runoffs (Fig. 1). Atmospheric deposition may also contribute to this high pyrogenic fingerprint.

\section{Link and partitioning between particulate and dissolved PAHs}

A quite high contribution of LMW and alkylated compounds (petrogenic signatures) is found for particulate PAHs, especially for some $G^{\prime} 1$ and $G^{\prime} 2$ stations, and, to a lower extent, for $G^{\prime} 3$ and G'4 stations. This could reflect the sorption of dissolved LMW PAHs to phytoplankton (Dachs and Méjanelle 2010; Del Vento and Dachs 2002; Gobas et al. 1999). Indeed, dissolved PAHs may be adsorbed to phytoplankton cells and enter phytoplankton membranes through passive diffusion (Del Vento and Dachs 2002). This phytoplankton uptake (adsorption and absorption) is considered as a key process in the transport, occurrence and distribution of particulate PAHs in the marine environment (Baker et al. 1991; Berrojalbiz et al. 2011; Duran and Cravo-Laureau 2016; Lipiatou et al. 1997). This assumption is reinforced by the positive correlation observed between LMW PAH and $n-\mathrm{C}_{17}$ concentrations (Table 2). In addition, the coupling and possible mobility between particulate and dissolved PAHs is observable through the positive significant correlation between the concentration of particulate PAHs (this study) and the concentration of dissolved PAHs (data issued from Fourati et al. 2017) $(r=0.41, n=29, p<0.05)$. Besides, the high TChl $a$, POC, PON, DOC and $\mathrm{PO}_{4}{ }^{3-}$ concentrations, as well as $\mathrm{AH}$ biogenic signatures recorded in the Sfax coastal area, in particular on its southern coast, reveals an elevated phytoplankton biomass and eutrophic conditions that may stimulate the sorption of LMW PAHs onto biogenic particles (Fig. S4). It should be noticed that the coupling between $n$ $\mathrm{C}_{17}$ and LMW PAHs might also mimic a possible natural origin of PAHs from the OM turnover or pigment degradation, as mentioned by Nizzetto et al. (2008). In contrast, the pyrogenic PAH signatures (dominance of HMWs) would be rather related to the adsorption of these compounds onto atmospheric/abiotic particles with inputs from wadis, surface runoff and sediment remobilisation in the Sfax coastal area, atmospheric particle deposition in the Mahrès area and a mixture of all these in the Gabès coastal area.

To better understand the partitioning of PAHs between the dissolved and particulate phases in the Gulf of Gabès, we determined the POC-water partition coefficients of PAHs $\left(\mathrm{K}_{\mathrm{oc}}\right) . \mathrm{K}_{\mathrm{oc}}$ is calculated for each individual compound by dividing the concentration associated with $\mathrm{SPM}\left(\mathrm{C}_{\mathrm{s}}\right.$, weight/ weight; data from the present study) by the concentration in the dissolved phase $\left(\mathrm{C}_{\mathrm{w}}\right.$, weight/volume; data from Fourati et al. 2017). This ratio $\left(K_{d}\right)$ is then normalized by the POC fraction ( $f_{\mathrm{oc}}$, POC concentration/SPM concentration). We plotted our measured $\log \mathrm{K}_{\mathrm{oc}}$ for selective PAHs (Naph, Phe, Acy, Ace, Flu, Ant, Flt, Pyr, BaA, Chr, BbF, BkF, BeP, BaP, IP, DA and BP) against corresponding $\log \mathrm{K}_{\mathrm{ow}}$ (partition coefficients between octanol and water; values issued from Mackay et al. 1992) (Fig. 8). We observe a significant positive linear relationship of the form: $\log \mathrm{K}_{\mathrm{oc}}=0.45 \log \mathrm{K}_{\mathrm{ow}}+3.29$ 
$(r=0.71, n=16, p<0.05)$. This equation reveals that the linear free energy relationship is applicable and that the hydrophobicity of the concerned PAHs influences their partitioning between POC and water. This result is in accordance with previous studies (Deng et al. 2006; Fernandes et al. 1997; Gustafson and Dickhut 1997; Li et al. 2014). We also plotted the theoretical relationship between $\log \mathrm{K}_{\mathrm{oc}}$ and $\log$ $\mathrm{K}_{\mathrm{ow}}$ proposed by Means et al. (1980) (Fig. 8). Because the slope of our equation $(0.45)$ is $<1$, which is in agreement with several works as well (Deng et al. 2006; Li et al. 2014), most of our measured $\log \mathrm{K}_{\mathrm{oc}}$ are above the predicted $\log \mathrm{K}_{\mathrm{oc}}$ values from Karickhoff et al. (1979) and Means et al. (1980). This shows that the strength of retention in particles is higher for LMW compounds (Bouloubassi and Saliot 1991; Fernandes et al. 1997), whereas measured $\log \mathrm{K}_{\mathrm{oc}}$ of HMW PAHs are closer to theoretical $\log \mathrm{K}_{\mathrm{oc}}$ values. This could be explained by the fact that LMW PAHs tend to preferentially sorb to biogenic particles, as stated above. This scavenging by biogenic particles is favoured for LMW PAHs and less efficient for HMW PAHs whose distribution is rather controlled by combustion (soot) particles, which limits their interactions with phytoplankton-derived particulate OM (Bouloubassi et al. 2006; Dachs et al. 1996). Hence, the sorption of LMW PAHs to biogenic particles in this phytoplankton-rich coastal area may account for the decoupling between LMW and HMW PAHs in their relationships between their $\log \mathrm{K}_{\mathrm{oc}}$ and $\log \mathrm{K}_{\mathrm{ow}}$. The partitioning of PAHs between POC and water would be thus driven by both hydrophobicity and the quality of particulate OM (i.e. high contribution of biogenic particles).

\section{Effect of rainfall on the distribution of particulate hydrocarbons}

For this study, the load factors after rainfalls for particulate $R$ and PAHs $_{-\mathrm{P}}$ concentrations varied from 2 to 23.5 and from 0.96 to 11.6, respectively. Guigue et al. (2011) reported lower factors for particulate $R$ and PAHs $_{-\mathrm{P}}$ in the Marseilles coastal area (0.36-1.4 and 0.87-2.0, respectively). On the contrary, Lim et al. (2007) indicated higher load factors for PAHs_p of the Singapore subsurface waters (0.7-38.5). Sfax is characterized by a semi-arid climate with annual average precipitations of $237 \mathrm{~mm}$. Even though precipitation events are very scarce and of low intensity during the year (Daoud and Dahech 2012), they allow the leaching of roads and the atmospheric washout, and thus the drainage of important amounts of pollutants, accumulated during long periods of time, to the coastal marine environment. This may explain the high concentrations (the highest PAH levels) and enrichment in hydrocarbons encountered at stations M3 $3_{-\mathrm{AR}}, \mathrm{M} 4_{-\mathrm{AR}}, \mathrm{M} 8_{-\mathrm{AR}}$ and $\mathrm{M} 12_{-\mathrm{AR}}$.

$\mathrm{AH}$ molecular profiles and ratios after rain indicated an input of petrogenic hydrocarbons at $\mathrm{M} 3_{-\mathrm{AR}}$ and $\mathrm{M} 4_{-\mathrm{AR}}$, while enrichment in biogenic hydrocarbons occurred at M8-AR and M12-AR (Fig. 6a-d). In addition, all stations present a higher

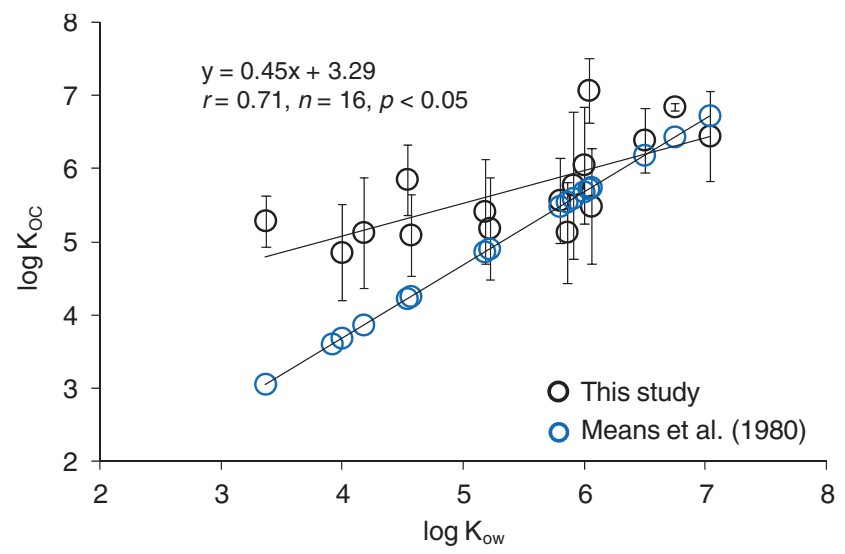

Fig. 8 Relationship between $\log \mathrm{K}_{\mathrm{ow}}$, reported by Mackay et al. (1992), and $\log \mathrm{K}_{\mathrm{oc}}$ determined in this study (black circles), and the theoretical relationship between $\log \mathrm{K}_{\mathrm{ow}}$ and $\log \mathrm{K}_{\mathrm{oc}}$ proposed by Means et al. (1980) (blue circles). Standard deviations and mean values of $\log \mathrm{K}_{\mathrm{oc}}$ are given for $n=6-22$, depending on the compound

terrigenous contribution (Ter/aq), especially at M3 $3_{-A R}$ and M12 -AR (Table 1). These patterns show that road run-offs with an accumulation in the $\mathrm{PK}_{4}$ outfall and the washout of harbours very likely represent the petrogenic sources for M3-AR and M4-AR. For M8 $8_{-A R}$ and M12 ${ }_{-A R}$, biogenic enrichment with higher terrestrial inputs and higher phytoplankton contributions $\left(n-\mathrm{C}_{15}\right)$ may be related to the Sidi Salem channel that carries nutrients and biological material to the southern Sfax area. The PAH molecular profiles after rain are very similar to those recorded before rain (Fig. 7a-d). The rain input at M3. $\mathrm{AR}$ and M4 $4_{-\mathrm{AR}}$ seems to increase petrogenic contributions, with an enrichment in alkylated Phe/Ant, as well as pyrogenic contributions, with mainly enrichment in Pyr and alkylated Flt/Pyr, leading to a decrease in LMW/HMW $-\mathrm{P}+\mathrm{A}$ and Flt/ (Flt + Pyr) (Fig. 7a, b). Station M8 $8_{-A R}$ is marked by petrogenic inputs (decrease in Flt/(Flt + Pyr)) with an enrichment in alkylated Naph and Flu and the preservation of the significant alkylated Phe/Ant contributions (Fig. 7c). HMW PAHs are known to be dominant in the atmospheric particulate (aerosols) phase, while LMW PAHs are dominant in the atmospheric gaseous phase. PAHs entering marine waters via the atmospheric gaseous phase are present at the dissolved state. Wet depositions (rains) lead to the deposition of both particulate and gaseous phases (Lipiatou et al. 1997). Therefore, the inputs of both petrogenic and pyrogenic particulate materials after rain at $\mathrm{M} 3_{-\mathrm{AR}}$ and $\mathrm{M} 4_{-\mathrm{AR}}$ may be due to the inputs of particulate HMW PAHs from aerosols and of dissolved LMW PAHs (such as phenanthrene) from the gaseous phase that are subsequently sorbed to (biogenic) marine particles. Besides, the road runoffs may also be a significant contributor to the input of LMW PAHs, especially in M8 $-\mathrm{AR}$ through the Sidi Salem channel. The PAH molecular profile of M12 ${ }_{\text {AR }}$ is similar to that of M12 (Fig. 7d), indicating that atmospheric deposition would be a significant source of hydrocarbons (mainly pyrogenic PAHs) in the Mahrès 
delegation. This pyrogenic input, also seen from Flt/(Flt + Pyr) values, highlights the potential influence of burning gas from the British Gas plant in this zone.

\section{Conclusion}

The present study reports for the first time the spatial distribution of particulate hydrocarbons in the surface waters of the Gulf of Gabès. Concentration levels indicate that the Gulf of Gabès is moderately impacted by particulate hydrocarbons. AHs and PAHs display similar repartitions, with the highest concentrations observed in the vicinity of harbours, channels and wadis or petrochemical activity areas. In these stations, petrogenic hydrocarbons dominate. We also observe the presence of pyrogenic (HMW) PAHs, which reflects combustion inputs. In the Sfax coastal area, hydrocarbon concentrations, as well as Alk/Par ratios, decrease within a coast-open sea gradient. This may underline the occurrence of weathering processes, such as biodegradation, which would contribute to the removal of hydrocarbons, especially the LMW compounds, off the coast. On the contrary, in the GabèsGhannouch area, hydrocarbon content does not decrease from coast to open sea. This may imply lower biodegradation processes due to a less eutrophicated system, as indicated by biogeochemical data. The partitioning of PAHs between the dissolved and particulate phases in the Gulf of Gabès seems to be controlled by hydrophobicity and OM nature. LMW PAHs exhibit a higher retention capacity in POC compared to HMW PAHs. This could be due to the fact that LMW compounds are preferentially sorbed to biogenic/sinking particles, i.e. phytoplankton and faecal pellets. The biogenic fingerprint of AHs and biogeochemical data suggest indeed that this environment is enriched in particulate biogenic organic matter favouring the transfer of LMW PAHs from the dissolved phase to the particulate phase. Interestingly, we found that in this semi-arid environment, rainfall events significantly increase the hydrocarbon concentrations in seawater (up to 23 times), due to the accumulation of pollutants for long periods of time. Thereby, inputs from surface runoffs, wadis and channels are considered as substantial sources of anthropogenic hydrocarbons in the coastal area of the Gulf of Gabès.

Acknowledgements We acknowledge the "Institut de recherche pour le développement" (IRD) for providing scholarship to R. Fourati through the "Allocations de Recherche pour une Thèse au Sud" (ARTS) program, as well as the Tunisian Ministry of Higher Education and Scientific Research for its financial support. This study was carried out in the framework of the IRD Action South project "MANGA" and the IRD French-Tunisian International Joint Laboratory (LMI) "COSYS-Med", and is a contribution to the WP3 C3A-Action MERMEX/MISTRALS. We are grateful to Z. Drira from the laboratory of Biodiversity and Aquatic Ecosystems (Faculty of Sciences, University of Sfax), as well as H. Sahnoun and T. Omar for their technical help during cruises. We thank the core parameter analytical platform (PAPB) of the Mediterranean
Institute of Oceanography (MIO) for performing biogeochemical analyses, and R. Sempéré for the use of the Agilent GC-MS.

\section{References}

Adhikari PL, Maiti K, Overton EB (2015) Vertical fluxes of polycyclic aromatic hydrocarbons in the northern Gulf of Mexico. Mar Chem 168:60-68. https://doi.org/10.1016/j.marchem.2014.11.001

Akkanen J, Tuikka A, Kukkonen JVK (2012) On the borderline of dissolved and particulate organic matter: partitioning and bioavailability of polycyclic aromatic hydrocarbons. Ecotoxicol Environ Saf 78: 91-98. https://doi.org/10.1016/j.ecoenv.2011.11.010

Alaya-Ltifi L, Selmi S (2014) Passerine abundance and diversity in a polluted oasis habitat in south-eastern Tunisia. Eur J Wildl Res 60: 535-541. https://doi.org/10.1007/s10344-014-0817-0

Aloulou F, Kallel M, Dammak M, Elleuch B, Saliot A (2010) Evennumbered $n$-alkanes $/ \mathrm{n}$-alkenes predominance in surface sediments of Gabes Gulf in Tunisia. Environ Earth Sci 61:1-10. https://doi.org/ 10.1007/s12665-009-0315-y

Aloulou F, Elleuch B, Kallel M (2012) Benthic foraminiferal assemblages as pollution proxies in the northern coast of Gabes Gulf, Tunisia. Environ Monit Assess 184:777-795. https://doi.org/10.1007/ s10661-011-2001-2

Amorri J, Geffroy-Rodier C, Boufahja F, Mahmoudi E, Aïssa P, Ksibi M, Amblès A (2011) Organic matter compounds as source indicators and tracers for marine pollution in a western Mediterranean coastal zone. Environ Sci Pollut Res 18:1606-1616. https://doi.org/10. 1007/s11356-011-0522-4

Asia L, Mazouz S, Guiliano M, Doumenq P, Mille G (2009) Occurrence and distribution of hydrocarbons in surface sediments from Marseille Bay (France). Mar Pollut Bull 58:443-451. https://doi. org/10.1016/j.marpolbul.2008.11.022

Azri C, Tili A, Serbaji MM, Medhioub K (2002) Etude des résidus de combustion des fuels liquide et solide et de traitement chimique du phosphate brut dans la ville de Sfax (Tunisie). Pollut Atm 44:297308

Baker JE, Eisenreich SJ, Eadie BJ (1991) Sediment trap fluxes and benthic recycling of organic carbon, polycyclic aromatic hydrocarbons, and polychlorobiphenyl congeners in Lake Superior. Environ Sci Technol 25:500-509. https://doi.org/10.1021/es00015a019

Béjaoui B, Rais S, Koutitonsky V (2004) Modélisation de la dispersion du phosphogypse dans le golfe de Gabès. Bull Inst Nat Sci Tech Mer Salammbô 31:103-109

Bel Hassen M, Drira Z, Hamza A, Ayadi H, Akrout F, Issaoui H (2008) Summer phytoplankton pigments and community composition related to water mass properties in the Gulf of Gabes. Estuar Coast Shelf Sci 77:645-656. https://doi.org/10.1016/j.ecss.2007.10.027

Bel Hassen M, Drira Z, Hamza A, Ayadi H, Akrout F, Messaoudi S, Issaoui H, Aleya L, Bouaïn A (2009) Phytoplankton dynamics related to water mass properties in the Gulf of Gabes: ecological implications. J Mar Syst 75:216-226. https://doi.org/10.1016/j. jmarsys.2008.09.004

Ben Amor R, Gueddari M (2016) Major ion geochemistry of Ghannouch-Gabes coastline (at Southeast Tunisia, Mediterranean Sea): study of the impact of phosphogypsum discharges by geochemical modeling and statistical analysis. Environ Earth Sci 75: 851. https://doi.org/10.1007/s12665-016-5666-6

Berrojalbiz N, Dachs J, Ojeda MJ, Valle MC, Castro-Jiménez J, Wollgast J, Ghiani M, Hanke G, Zaldivar JM (2011) Biogeochemical and physical controls on concentrations of polycyclic aromatic hydrocarbons in water and plankton of the Mediterranean and Black Seas. Glob Biogeochem Cycles 25:1-14. https://doi.org/10.1029/ 2010GB003775 
Bligh E, Dyer W (1959) A rapid method of total lipid extraction and purification. Can J Biochem Physiol 37:911-917

Blumer M, Guillard RRL, Chase T (1971) Hydrocarbons of marine phytoplankton. Mar Biol 8:183-189. https://doi.org/10.1007/ BF00355214

Bouloubassi I, Saliot A (1991) Composition and sources of dissolved and particulate PAH in surface waters from the Rhone delta (NW Mediterranean). Mar Pollut Bull 22:588-594. https://doi.org/10. 1016/0025-326X(91)90246-O

Bouloubassi I, Saliot A (1993) Dissolved, particulate and sedimentary naturally derived polycyclic aromatic hydrocarbons in a coastal environment: geochemical significance. Mar Chem 42:127-143. https://doi.org/10.1016/0304-4203(93)90242-G

Bouloubassi I, Méjanelle L, Pete R, Fillaux J, Lorre A, Point V (2006) PAH transport by sinking particles in the open Mediterranean Sea: a 1 year sediment trap study. Mar Pollut Bull 52:560-571. https://doi. org/10.1016/j.marpolbul.2005.10.003

Bourbonniere RA, Meyers PA (1996) Sedimentary geolipid records of historical changes in the watersheds and productivities of Lakes Ontario and Erie. Limnol Oceanogr 41:352-359. https://doi.org/ 10.4319/lo.1996.41.2.0352

Broman D, Naef C, Rolff C, Zebuehr Y (1991) Occurrence and dynamics of polychlorinated dibenzo-p-dioxins and dibenzofurans and polycyclic aromatic hydrocarbons in the mixed surface layer of remote coastal and offshore waters of the Baltic. Environ Sci Technol 25: 1850-1864. https://doi.org/10.1021/es00023a002

Budzinski H, Jones I, Bellocq J, Piérard C, Garrigues P (1997) Evaluation of sediment contamination by polycyclic aromatic hydrocarbons in the Gironde estuary. Mar Chem 58:85-97. https://doi.org/10.1016/ S0304-4203(97)00028-5

Castro-Jiménez J, Berrojalbiz N, Wollgast J, Dachs J (2012) Polycyclic aromatic hydrocarbons (PAHs) in the Mediterranean Sea: atmospheric occurrence, deposition and decoupling with settling fluxes in the water column. Environ Pollut 166:40-47. https://doi.org/10. 1016/j.envpol.2012.03.003

Chen J, Henderson G, Grimm CC, Lloyd SW, Laine RA (1998) Termites fumigate their nests with naphthalene. Nature 392:558-559. https:// doi.org/10.1038/33305

Cincinelli A, Stortini AM, Perugini M, Checchini L, Lepri L (2001) Organic pollutants in sea-surface microlayer and aerosol in the coastal environment of Leghorn - (Tyrrhenian Sea). Mar Chem 76:77-98. https://doi.org/10.1016/S0304-4203(01)00049-4

Cincinelli A, Martellini T, Bittoni L, Russo A, Gambaro A, Lepri L (2008) Natural and anthropogenic hydrocarbons in the water column of the Ross Sea (Antarctica). J Mar Syst 73:208-220. https:// doi.org/10.1016/j.jmarsys.2007.10.010

Colombo JC, Pelletier E, Brochu C, Khallil M, Catoggio JA (1989) Determination of hydrocarbon sources using n-alkane and polyaromatic hydrocarbon distribution indexes. Case study: Rio de la Plata estuary, Argentina. Environ Sci Technol 23:888-894. https://doi.org/10.1021/es00065a019

Commendatore MG, Esteves JL (2004) Natural and anthropogenic hydrocarbons in sediments from the Chubut River (Patagonia, Argentina). Mar Pollut Bull 48:910-918. https://doi.org/10.1016/j. marpolbul.2003.11.015

Commendatore MG, Nievas ML, Amin O, Esteves JL (2012) Sources and distribution of aliphatic and polyaromatic hydrocarbons in coastal sediments from the Ushuaia Bay (Tierra del Fuego, Patagonia, Argentina). Mar Environ Res 74:20-31. https://doi.org/ 10.1016/j.marenvres.2011.11.010

Cripps GC (1989) Problems in the identification of anthropogenic hydrocarbons against natural background levels in the Antarctic. Antarct Sci 1:307-312. https://doi.org/10.1017/S0954102089000465

Dachs J, Mejanelle L (2010) Organic pollutants in coastal waters, sediments, and biota: a relevant driver for ecosystems during the anthropocene? Estuar Coasts 33:1-14. https://doi.org/10.1007/ s12237-009-9255-8

Dachs J, Bayona JM, Fowler SW, Miquel J-C, Albaigés J (1996) Vertical fluxes of polycyclic aromatic hydrocarbons and organochlorine compounds in the western Alboran Sea (southwestern Mediterranean). Mar Chem 52:75-86

Dachs J, Bayona JM, Fillaux J, Saliot A, Albaiges J (1999) Evaluation of anthropogenic and biogenic inputs into the western Mediterranean using molecular markers. Mar Chem 65:195-210

Dachs J, Lohmann R, Ockenden WA, Méjanelle L, Eisenreich SJ, Jones KC (2002) Oceanic biogeochemical controls on global dynamics of persistent organic pollutants. Environ Sci Technol 36:4229-4237. https://doi.org/10.1021/es025724k

Daoud A, Dahech S (2012) Résilience de l'agglomération de Sfax (Tunisie méridionale) face au changement climatique: essai d'évaluation, Climatologie, numéro spécial "Climats et changement climatique dans les villes.” 109-126. doi:https://doi.org/10.4267/ climatologie. 738

Dauner ALL, Hernández EA, MacCormack WP, Martins CC (2015) Molecular characterisation of anthropogenic sources of sedimentary organic matter from Potter Cove, King George Island, Antarctica. Sci Total Environ 502:408-416. https://doi.org/10.1016/j.scitotenv. 2014.09.043

Del Vento S, Dachs J (2002) Prediction of uptake dynamics of persistent organic pollutants by bacteria and phytoplankton. Environ Toxicol Chem 21:2099-2107. https://doi.org/10.1002/etc.5620211013

Deng H, Peng P, Huang W, Song J (2006) Distribution and loadings of polycyclic aromatic hydrocarbons in the Xijiang River in Guangdong, South China. Chemosphere 64:1401-1411. https:// doi.org/10.1016/j.chemosphere.2005.12.027

DGEQV (2008) Etude de dépollution et de réhabilitation des côtes sud de Sfax. PHASE-1 : Inventaire et caractérisation des sources de pollution. Note de synthese. Ministère de l'Environnement et du Développement Durable, Sfax

Douglas AG, Eglinton G (1966) The distribution of n-alkane. In: Swain T (ed) Comparative Phytochemistry. Academic Press, London, pp 5778

Drira Z, Elloumi J, Guermazi W, Bel Hassen M, Hamza A, Ayadi H (2014) Seasonal changes on planktonic diatom communities along an inshore-offshore gradient in the Gulf of Gabes (Tunisia). Acta Ecol Sin 34:34-43. https://doi.org/10.1016/j.chnaes.2013.11.005

Drira Z, Kmiha-Megdiche S, Sahnoun H, Hammami A, Allouche N, Tedetti M, Ayadi H (2016) Assessment of anthropogenic inputs in the surface waters of the southern coastal area of Sfax during spring (Tunisia, Southern Mediterranean Sea). Mar Pollut Bull 104:355363. https://doi.org/10.1016/j.marpolbul.2016.01.035

Duran R, Cravo-Laureau C (2016) Role of environmental factors and microorganisms in determining the fate of polycyclic aromatic hydrocarbons in the marine environment. FEMS Microbiol Rev 40: 814-830. https://doi.org/10.1093/femsre/fuw031

Eggleton J, Thomas KV (2004) A review of factors affecting the release and bioavailability of contaminants during sediment disturbance events. Environ Int 30:973-980. https://doi.org/10.1016/j.envint. 2004.03.001

El Nemr A, Abd-Allah AM (2003) Contamination of polycyclic aromatic hydrocarbons (PAHs) in microlayer and subsurface waters along Alexandria coast, Egypt. Chemosphere 52:1711-1716. https://doi. org/10.1016/S0045-6535(03)00300-X

Fernandes MB, Sicre MA, Boireau A, Tronczynski J (1997) Polyaromatic hydrocarbon $(\mathrm{PAH})$ distributions in the Seine River and its estuary. Mar Pollut Bull 34:857-867. https://doi.org/10.1016/S0025$326 \mathrm{X}(97) 00063-5$

Fourati R, Tedetti M, Guigue C, Goutx M, Garcia N, Zaghden H, Sayadi S, Elleuch B (2017) Sources and spatial distribution of dissolved aliphatic and polycyclic aromatic hydrocarbons in surface coastal 
waters of the Gulf of Gabès (Tunisia, Southern Mediterranean Sea). Prog Oceanogr. https://doi.org/10.1016/j.pocean.2017.02.001

Gobas FAPC, Wilcockson JB, Russell RW, Haffner GD (1999) Mechanism of biomagnification in fish under laboratory and field conditions. Environ Sci Technol 33:133-141. https://doi.org/10. $1021 / \mathrm{es} 980681 \mathrm{~m}$

Goutx M, Saliot A (1980) Relationship between dissolved and particulate fatty acids and hydrocarbons, chlorophyll a and zooplankton biomass in Villefranche Bay, Mediterranean Sea. Mar Chem 8:299-318

Guigue C, Tedetti M, Giorgi S, Goutx M (2011) Occurrence and distribution of hydrocarbons in the surface microlayer and subsurface water from the urban coastal marine area off Marseilles, Northwestern Mediterranean Sea. Mar Pollut Bull 62:2741-2752. https://doi.org/10.1016/j.marpolbul.2011.09.013

Guigue C, Bigot L, Turquet J, Tedetti M, Ferretto N, Goutx M, Cuet P (2015) Hydrocarbons in a coral reef ecosystem subjected to anthropogenic pressures (La Réunion Island, Indian Ocean ). Environ Chem 12:350-365. https://doi.org/10.1071/en14194

Gustafson KE, Dickhut RM (1997) Distribution of polycyclic aromatic hydrocarbons in southern Chesapeake Bay surface water: evaluation of three methods for determining freely dissolved water concentrations. Environ Toxicol Chem 16:452-461. https://doi.org/10.1002/ etc. 5620160310

Gustafsson Ö, Nilsson N, Bucheli TD (2001) Dynamic colloid-water partitioning of pyrene through a coastal Baltic spring bloom. Environ Sci Technol 35:4001-4006. https://doi.org/10.1021/ es0003019

Herbes SE (1977) Partitioning of polycyclic aromatic hydrocarbons between dissolved and particulate phases in natural waters. Water Res $11: 493-496$

Hylland K (2006) Polycyclic aromatic hydrocarbon (PAH) ecotoxicology in marine ecosystems. J Toxicol Environ Heal Part A 69:109-123. https://doi.org/10.1080/15287390500259327

Karickhoff SW, Brown DS, Scott TA (1979) Sorption of hydrophobic pollutants on natural sediments. Water Res 13:241-248

Katsoyiannis A, Breivik K (2014) Model-based evaluation of the use of polycyclic aromatic hydrocarbons molecular diagnostic ratios as a source identification tool. Environ Pollut 184:488-494. https://doi. org/10.1016/j.envpol.2013.09.028

Katsoyiannis A, Terzi E, Cai QY (2007) On the use of PAH molecular diagnostic ratios in sewage sludge for the understanding of the PAH sources. Is this use appropriate? Chemosphere 69:1337-1339. https://doi.org/10.1016/j.chemosphere.2007.05.084

Kennish MJ (1992) Ecology of estuaries: anthropogenic effects. CRC Press

Ko F-C, Sanford LP, Baker JE (2003) Internal recycling of particle reactive organic chemicals in the Chesapeake Bay water column. Mar Chem 81:163-176. https://doi.org/10.1016/S0304-4203(03)000276

Li H, Lu L, Huang W, Yang J, Ran Y (2014) In-situ partitioning and bioconcentration of polycyclic aromatic hydrocarbons among water, suspended particulate matter, and fish in the Dongjiang and Pearl Rivers and the Pearl River Estuary, China. Mar Pollut Bull 83:306316. https://doi.org/10.1016/j.marpolbul.2014.04.036

Li P, Cao J, Diao X, Wang B, Zhou H, Han Q, Zhen P, Li Y (2015) Spatial distribution, sources and ecological risk assessment of polycyclic aromatic hydrocarbons in surface seawater from Yangpu Bay, China. Mar Pollut Bull 93:53-60. https://doi.org/10.1016/j. marpolbul.2015.02.015

Lim L, Wurl O, Karuppiah S, Obbard JP (2007) Atmospheric wet deposition of PAHs to the sea-surface microlayer. Mar Pollut Bull 54: 1212-1219. https://doi.org/10.1016/j.marpolbul.2007.03.023

Lipiatou E, Saliot A (1991) Fluxes and transport of anthropogenic and natural polycyclic aromatic hydrocarbons in the western Mediterranean Sea. Mar Chem 32:51-71. https://doi.org/10.1016/ 0304-4203(91)90025-R
Lipiatou E, Tolosa I, Simó R, Bouloubassi I, Dachs J, Marti S, Sicre MA, Bayona JM, Grimalt JO, Saliot A, Albaigés J (1997) Mass budget and dynamics of polycyclic aromatic hydrocarbons in the Mediterranean Sea. Deep Res Part II Top Stud Oceanogr 44:881905. https://doi.org/10.1016/S0967-0645(96)00093-8

Liu L, Wang J, Wei G, Guan Y, Zeng EY (2012) Polycyclic aromatic hydrocarbons (PAHs) in continental shelf sediment of China: implications for anthropogenic influences on coastal marine environment. Environ Pollut 167:155-162. https://doi.org/10.1016/j.envpol.2012. 03.038

Louati A, Elleuch B, Kallel M, Saliot A, Dagaut J, Oudot J (2001) Hydrocarbon contamination of coastal sediments from the Sfax area (Tunisia), Mediterranean Sea. Mar Pollut Bull 42:444-451. https:// doi.org/10.1016/S0025-326X(00)00179-X

Mackay D, Shiu WY, Ma KC (1992) Illustrated handbook of physicalchemical properties and environmental fate for organic chemicals. Lewis Publishers

Maldonado C, Bayona JM, Bodineau L (1999) Sources, distribution, and water column processes of aliphatic and polycyclic aromatic hydrocarbons in the Northwestern Black Sea water. Environ Sci Technol 33:2693-2702. https://doi.org/10.1021/es9811647

Means JC, Wood SG, Hassett JJ, Banwart WL (1980) Sorption of polynuclear aromatic hydrocarbons by sediments and soils. Environ Sci Technol 14:1524-1528. https://doi.org/10.1021/es60172a005

Mille G, Guiliano M, Asia L, Malleret L, Jalaluddin N (2006) Sources of hydrocarbons in sediments of the bay of fort de France (Martinique). Chemosphere 64:1062-1073. https://doi.org/10.1016/j. chemosphere.2005.12.001

Mille G, Asia L, Guiliano M, Malleret L, Doumenq P (2007) Hydrocarbons in coastal sediments from the Mediterranean sea (Gulf of Fos area, France). Mar Pollut Bull 54:566-575. https:// doi.org/10.1016/j.marpolbul.2006.12.009

Neff J (2002) Polycyclic aromatic hydrocarbons in the ocean. In: Neff J (ed) Bioaccumulation in marine organisms. Elsevier, Oxford, pp 241-318

Nizzetto L, Lohmann R, Gioia R, Jahnke A, Temme C, Dachs J, Herckes P, Di Guardo A, Jones KC (2008) PAHs in air and seawater along a North-South Atlantic transect: trends, processes and possible sources. Environ Sci Technol 42:1580-1585. https://doi.org/10. 1021/es0717414

Readman JW, Fillmann G, Tolosa I, Bartocci J, Villeneuve JP, Catinni C, Mee LD (2002) Petroleum and PAH contamination of the Black Sea. Mar Pollut Bull 44:48-62. https://doi.org/10.1016/S0025-326X(01) 00189-8

Rekik A, Denis M, Dugenne M, Barani A, Maalej S, Ayadi H (2014) Seasonal distribution of ultraphytoplankton and heterotrophic prokaryotes in relation to abiotic variables on the north coast of Sfax after restoration. Mar Pollut Bull 84:280-305. https://doi.org/10. 1016/j.marpolbul.2014.05.003

Rielley G, Collier RJ, Jones DM, Eglinton G (1991) The biogeochemistry of Ellesmere Lake, U.K.-I: source correlation of leaf wax inputs to the sedimentary lipid record. Org Geochem 17:901-912. https://doi. org/10.1016/0146-6380(91)90031-E

Shang J, Chen J, Shen Z, Xiao X, Yang H, Wang Y, Ruan A (2015) Photochemical degradation of PAHs in estuarine surface water: effects of DOM, salinity, and suspended particulate matter. Environ Sci Pollut Res 22:12374-12383. https://doi.org/10.1007/s11356015-4543-2

Shrestha G, Traina SJ, Swanston CW (2010) Black Carbon's properties and role in the environment: a comprehensive review. Sustainability 2:294-320. https://doi.org/10.3390/su2010294

Tobiszewski M, Namieśnik J (2012) PAH diagnostic ratios for the identification of pollution emission sources. Environ Pollut 162:110 119. https://doi.org/10.1016/j.envpol.2011.10.025

Tornero V, Hanke G (2016) Chemical contaminants entering the marine environment from sea-based sources: a review with a focus on 
European seas. Mar Pollut Bull 112:17-38. https://doi.org/10.1016/ j.marpolbul.2016.06.091

Trabelsi A, Saad M, Masmoudi M, Alfaro SC (2015) Atmospheric aerosols and their impact on surface solar irradiation in Kerkennah Islands (eastern Tunisia). Atmos Res 161-162:102-107. https:// doi.org/10.1016/j.atmosres.2015.04.006

Trabelsi A, Masmoudi M, Quisefit JP, Alfaro SC (2016) Compositional variability of the aerosols collected on Kerkennah Islands ( central Tunisia). Atmos Res 169:292-300

Tsapakis M, Apostolaki M, Eisenreich S, Stephanou EG (2006) Atmospheric deposition and marine sedimentation fluxes of polycyclic aromatic hydrocarbons in the Eastern Mediterranean Basin. Environ Sci Technol 40:4922-4927. https://doi.org/10.1021/ es $060487 \mathrm{x}$

Volkman JK, Holdsworth DG, Neill GP, Bavor HJ Jr (1992) Identification of natural, anthropogenic and petroleum hydrocarbons in aquatic sediments. Sci Total Env 112:203-219. https://doi.org/10.1016/ 0048-9697(92)90188-X

Wang Z, Fingas MF (2003) Development of oil hydrocarbon fingerprinting and identification techniques. Mar Pollut Bull 47:423-452. https://doi.org/10.1016/S0025-326X(03)00215-7

Wang Z, Fingas M, Landriault M, Sigouin L, Feng Y, Mullin J (1997) Using systematic and comparative analytical data to identify the source of an unknown oil on contaminated birds. J Chromatogr A 775:251-265. https://doi.org/10.1016/S0021-9673(97)00298-7

Wang Z, Fingas M, Shu YY, Sigouin L, Landriault M, Lambert P, Turpin R, Campagna P, Mullin J (1999) Quantitative characterization of PAHs in burn residue and soot samples and differentiation of pyrogenic PAH1 from petrogenic PAHs - the 1994 mobile burn study. Environ Sci Technol 33:3100-3109. https://doi.org/10.1021/ es990031y

Wilcke W, Krauss M, Amelung W (2002) Carbon isotope signature of polycyclic aromatic hydrocarbons (PAHs): evidence for different sources in tropical and temperate environments? Environ Sci Technol 36:3530-3535
Witt G (2002) Occurrence and transport of polycyclic aromatic hydrocarbons in the water bodies of the Baltic Sea. Mar Chem 79:49-66. https://doi.org/10.1016/S0304-4203(02)00035-X

Wu Y-L, Wang X-H, Li Y-Y, Hong H-S (2011) Occurrence of polycyclic aromatic hydrocarbons (PAHs) in seawater from the Western Taiwan Strait, China. Mar Pollut Bull 63:459-463. https://doi.org/ 10.1016/j.marpolbul.2011.03.008

Youngblood WW, Blumer M (1975) Polycyclic aromatic hydrocarbons in the environment: homologous series in soils and recent marine sediments. Geochim Cosmochim Acta 188:53-55. https://doi.org/10. 1126/science. 188.4183 .53

Yunker MB, Macdonald RW, Vingarzan R, Mitchell RH, Goyette D, Sylvestre S (2002) PAHs in the Fraser River basin: a critical appraisal of PAH ratios as indicators of PAH source and composition. Org Geochem 33:489-515. https://doi.org/10.1016/S0146-6380(02) 00002-5

Zaghden H, Kallel M, Louati A, Elleuch B, Oudot J, Saliot A (2005) Hydrocarbons in surface sediments from the Sfax coastal zone, (Tunisia) Mediterranean Sea. Mar Pollut Bull 50:1287-1294. https://doi.org/10.1016/j.marpolbul.2005.04.045

Zaghden H, Kallel M, Elleuch B, Oudot J, Saliot A (2007) Sources and distribution of aliphatic and polyaromatic hydrocarbons in sediments of Sfax, Tunisia, Mediterranean Sea. Mar Chem 105:70-89. https://doi.org/10.1016/j.marchem.2006.12.016

Zaghden H, Kallel M, Elleuch B, Oudot J, Saliot A, Sayadi S (2014) Evaluation of hydrocarbon pollution in marine sediments of Sfax coastal areas from the Gabes Gulf of Tunisia, Mediterranean Sea. Environ Earth Sci 72:1073-1082. https://doi.org/10.1007/s12665013-3023-6

Zaghden H, Tedetti M, Sayadi S, Serbaji MM, Elleuch B, Saliot A (2017) Origin and distribution of hydrocarbons and organic matter in the surficial sediments of the Sfax-Kerkennah channel (Tunisia, Southern Mediterranean Sea). Mar Pollut Bull 117:414-428. https://doi.org/10.1016/j.marpolbul.2017.02.007 